TRANSACTIONS OF THE

AMERICAN MATHEMATICAL SOCIETY

Volume 361, Number 5, May 2009, Pages 2567-2586

S 0002-9947(08)04789-2

Article electronically published on November 4, 2008

\title{
LOCAL WELL POSEDNESS, ASYMPTOTIC BEHAVIOR AND ASYMPTOTIC BOOTSTRAPPING FOR A CLASS OF SEMILINEAR EVOLUTION EQUATIONS OF THE SECOND ORDER IN TIME
}

\author{
A. N. CARVALHO AND J. W. CHOLEWA
}

\begin{abstract}
A class of semilinear evolution equations of the second order in time of the form $u_{t t}+A u+\mu A u_{t}+A u_{t t}=f(u)$ is considered, where $-A$ is the Dirichlet Laplacian, $\Omega$ is a smooth bounded domain in $\mathbb{R}^{N}$ and $f \in C^{1}(\mathbb{R}, \mathbb{R})$. A local well posedness result is proved in the Banach spaces $W_{0}^{1, p}(\Omega) \times W_{0}^{1, p}(\Omega)$ when $f$ satisfies appropriate critical growth conditions. In the Hilbert setting, if $f$ satisfies an additional dissipativeness condition, the nonlinear semigroup of global solutions is shown to possess a gradient-like attractor. Existence and regularity of the global attractor are also investigated following the unified semigroup approach, bootstrapping and the interpolation-extrapolation techniques.
\end{abstract}

\section{INTRODUCTORY NOTES}

In this article we consider a class of semilinear evolution equations of the second order in time of the form

$$
u_{t t}+A u+\mu A u_{t}+A u_{t t}=f(u), t>0,
$$

with the initial conditions

$$
u(0)=u_{0}, u_{t}(0)=v_{0}
$$

from a suitably chosen Banach space $X^{1}$. Here $-A: D(A) \subset X \rightarrow X$ is the generator of an exponentially decaying analytic semigroup of bounded linear operators in $X$ and $X^{1}$ is the domain of $A$ with the graph norm.

The equations that fall into this class are known to represent some sort of 'propagation problems' (see [4, 6, 22, also [19] and the references therein), among which a specific problem is

$$
\left\{\begin{array}{l}
u_{t t}-\Delta u-\mu \Delta u_{t}-\Delta u_{t t}=f(u), t>0, x \in \Omega, \\
u=0, t \geq 0, x \in \partial \Omega \\
u(0, x)=u_{0}(x), u_{t}(0, x)=v_{0}(x), x \in \Omega,
\end{array}\right.
$$

Received by the editors May 21, 2007.

2000 Mathematics Subject Classification. Primary 35G25, 35B33, 35B40, 35B41, 35B65.

Key words and phrases. Evolution equations of the second order in time, existence, uniqueness and continuous dependence of solutions on initial conditions, asymptotic behavior of solutions, attractors, regularity, critical exponents.

This research was partially supported by grant \# 300.889/92-5 CNPq and grant \# 03/10042-0 FAPESP, Brazil. 
where $\mu>0, \Omega$ is a bounded smooth domain in $\mathbb{R}^{N}$ and $f: \mathbb{R} \rightarrow \mathbb{R}$ is a locally Lipschitz function satisfying suitable growth and dissipativeness conditions.

Denote by $\lambda_{1}$ the first eigenvalue of $-\Delta$ with Dirichlet boundary conditions in $\Omega$.

In [19] the problem (1.3) was considered in the Hilbert space $H_{0}^{1}(\Omega) \times H_{0}^{1}(\Omega)$. There the authors prove the existence of a global attractor under the assumptions

$$
\lim \sup _{|s| \rightarrow \infty} \frac{f(s)}{s}<\lambda_{1},
$$

and

$$
\begin{aligned}
& |f(s)| \leq c\left(1+|s|^{\rho}\right), s \in \mathbb{R}, \text { where } \rho \in(1, \infty) \text { is } \\
& \text { arbitrary if } N=1,2 \text { and } \rho \leq \frac{N+2}{N-2} \text { if } N \geq 3 .
\end{aligned}
$$

The difficulties connected with the consideration of the critical exponent $\rho^{*}=\frac{N+2}{N-2}$ $(N \geq 3)$ have been successfully overcome following [14] and 21]. A key point in this consideration is to obtain the existence of an absorbing set accompanied by the suitable estimates for the solutions, from which the following asymptotic compactness property could be inferred:

(ACP) if $r>0,\left\|u_{0 n}\right\|_{H_{0}^{1}(\Omega)}+\left\|v_{0 n}\right\|_{H_{0}^{1}(\Omega)} \leq r$ and $t_{n} \nearrow \infty$, there is a subsequence $\left\{n_{k}\right\}$ so that $\left\{u\left(t_{n_{k}}, u_{0 n_{k}}, v_{0 n_{k}}\right)\right\}$ and $\left\{u_{t}\left(t_{n_{k}}, u_{0 n_{k}}, v_{0 n_{k}}\right)\right\}$ are convergent in $H_{0}^{1}(\Omega)$.

As for the existence-uniqueness results in 19, they have been quoted from previous references [17, 20, where the proof was based on the Faedo-Galerkin method.

In this paper we show that the semigroup approach, known from the geometric theory of semilinear problems (see [12, 15]) and dissipative systems theory (see [10, 13), is applicable to the problems that fall into the class (1.1)-(1.2) and provides the relevant information about their solutions.

Within this approach we obtain a local well posedness result for 1.3 in the Banach space $W_{0}^{1, p}(\Omega) \times W_{0}^{1, p}(\Omega), p \in(1, \infty)$, provided that one of the following conditions holds:

(H1) $\quad p>N$ and $f: \mathbb{R} \rightarrow \mathbb{R}$ is a locally Lipschitz continuous map.

(H2) $1<p<N$ and $f$ satisfies

(*) $\quad\left|f\left(s_{1}\right)-f\left(s_{2}\right)\right| \leq c\left|s_{1}-s_{2}\right|\left(1+\left|s_{1}\right|^{\rho-1}+\left|s_{2}\right|^{\rho-1}\right), s_{1}, s_{2} \in \mathbb{R}$, for some $c>0$ and $1<\rho \leq \frac{N+p}{N-p}$.

$$
1<p=N \text { and } f \text { satisfies }(*) \text { for some } c>0 \text { and } \rho \in(1, \infty) \text {. }
$$

Within the same approach we then obtain, for $p=2$ and assuming (1.4), a global well posedness result and show that the associated semigroup of global solutions has a global attractor which has the structure of the attractor of a gradient system (see [10]). 
For $p=2$ and in the subcritical case, that is, when

(SC) $N=1$ and $f: \mathbb{R} \rightarrow \mathbb{R}$ is a locally Lipschitz continuous function, or $N=2$ and $(*)$ in (1.7) is satisfied with some $\rho \in(1, \infty), c>0$, or $N \geq 3$ and $(*)$ in (1.7) holds with some $1<\rho<\frac{N+2}{N-2}, c>0$,

the mentioned approach provides, besides the existence of a global attractor $\mathbf{A}$, some additional regularity for the solutions in $\mathbf{A}$ through an asymptotic bootstrapping procedure.

In the case involving the critical exponent $\rho^{*}=\frac{N+2}{N-2}$, and specifically when

$$
N \geq 3, f \in C^{1}(\mathbb{R}, \mathbb{R}) \text { and }\left|f^{\prime}(s)\right| \leq c\left(1+|s|^{\frac{N+2}{N-2}-1}\right), s \in \mathbb{R},
$$

the existence of the global attractor is obtained with the aid of Alekseev's nonlinear variation of constants formula (see [2]). In either case the attractor is gradient-like (see [11]); that is,

$$
\mathbf{A}=W^{u}(\mathcal{E})
$$

where $\mathcal{E}$ denotes the set of equilibria of (1.3) and $W^{u}(\mathcal{E})$ is the unstable set of $\mathcal{E}$.

To be more specific about our technique and approach note first that in the variables $(t, v)$, with $v=(I+A) u$, the problem (1.1)-(1.2) becomes

$$
v_{t t}+\mu \Lambda v_{t}+\Lambda v=f^{e}(v), t>0,
$$

with the initial conditions

$$
v(0)=v_{0}, v_{t}(0)=w_{0}
$$

in a Banach space $X$, where $\Lambda:=I-(I+A)^{-1} \in L(X), f^{e}:=f \circ(I-\Lambda): X \rightarrow X$ is locally Lipschitz continuous, and $X:=X^{0}$ is defined as the extrapolation space of $X^{1}$ generated by the realization of $A$ in $X^{1}$ (see [1]).

In the extrapolation space $X$ the resemblance of (the counterpart (1.11)) of (1.1) with the classical strongly damped wave equation seems worthy to be mentioned and exploited. To exhibit these types of properties it is then reasonable to substitute $w:=v_{t}$, which leads to the first order problem

$$
\frac{d}{d t}\left[\begin{array}{c}
v \\
w
\end{array}\right]+\mathcal{A}_{\mu}\left[\begin{array}{c}
v \\
w
\end{array}\right]=\mathcal{F}\left(\left[\begin{array}{c}
v \\
w
\end{array}\right]\right), t>0, \quad\left[\begin{array}{c}
v \\
w
\end{array}\right]_{\left.\right|_{t=0}}=\left[\begin{array}{c}
v_{0} \\
w_{0}
\end{array}\right]
$$

in variables $(t, v, w)$ with $\mathcal{A}_{\mu} \in L(X \times X)$ given in the matrix form as

$$
\mathcal{A}_{\mu}:=\left[\begin{array}{cc}
0 & -I \\
\Lambda & \mu \Lambda
\end{array}\right]=\left[\begin{array}{cc}
0 & -I \\
I-(I+A)^{-1} & \mu\left(I-(I+A)^{-1}\right)
\end{array}\right]
$$

and a locally Lipschitz map $\mathcal{F}: X \times X \rightarrow X \times X$ being defined by

$$
\mathcal{F}\left(\left[\begin{array}{l}
v \\
w
\end{array}\right]\right)=\left[\begin{array}{c}
0 \\
f^{e}(v)
\end{array}\right],\left[\begin{array}{l}
v \\
w
\end{array}\right] \in X \times X .
$$

In the above setting the linear operator $\mathcal{A}_{\mu}$ defined in the product (extrapolation) space $X \times X$ essentially resembles the strongly damped wave operator appearing in the context of the classical wave equations (see e.g. [5, 8, 18]). The implicit conclusion drawn from the above generalities is that the problem (1.1) should be well posed in the Banach spaces $W_{0}^{1, p}(\Omega) \times W_{0}^{1, p}(\Omega), p \in(1, \infty)$, and that its solutions should exhibit some smoothing properties at least at infinity and at least in the (subcritical) case (SC). Also the existence of an attractor should follow within the semigroup approach as for the problems enjoying a gradient-like structure. 
To be more specific about our results, let $A$ be the negative Dirichlet Laplacian $\Delta$ in $L^{p}(\Omega), p \in(1, \infty)$, with the domain $W^{2, p}(\Omega) \cap W_{0}^{1, p}(\Omega)$ and consider its closed extension 11 to $X=\left(W_{0}^{1, p^{\prime}}(\Omega)\right)^{\prime}=: W^{-1, p}(\Omega)$.

As for the local well posedness of (1.1)-(1.2) we then obtain the following theorem.

Theorem 1.1. For each $u_{0}, v_{0} \in W_{0}^{1, p}(\Omega)$, there exists $\tau\left(u_{0}, v_{0}\right)>0$ and a unique function $u(\cdot) \in C^{2}\left(\left[0, \tau\left(u_{0}, v_{0}\right)\right), W_{0}^{1, p}(\Omega) \times W_{0}^{1, p}(\Omega)\right)$ satisfying

$$
u(0)=u_{0}, u_{t}(0)=v_{0},
$$

and

$$
u_{t t}(t)+\mu A u_{t}(t)+A u(t)+A u_{t t}(t)=f(u(t)) \text { for each } t \in\left[0, \tau\left(u_{0}, v_{0}\right)\right)
$$

provided that one of the conditions (H1), (H2) or (H3) in (1.7) holds.

Furthermore, functions $u\left(t, u_{0}, v_{0}\right)$ and $u_{t}\left(t, u_{0}, v_{0}\right)$ depend continuously on its variables $\left(t, u_{0}, v_{0}\right) \in\left[0, \tau\left(u_{0}, v_{0}\right)\right) \times W_{0}^{1, p}(\Omega) \times W_{0}^{1, p}(\Omega)$ in the norm of $W_{0}^{1, p}(\Omega) \times$ $W_{0}^{1, p}(\Omega)$ and either $\tau_{u_{0}, v_{0}}=\infty$ or $\left\|u\left(t, u_{0}, v_{0}\right)\right\|_{W_{0}^{1, p}(\Omega)}+\left\|u_{t}\left(t, u_{0}, v_{0}\right)\right\|_{W_{0}^{1, p}(\Omega)} \rightarrow \infty$ as $t \rightarrow \tau_{u_{0}, v_{0}}^{-}$.

As for the global well posedness result in $H_{0}^{1}(\Omega) \times H_{0}^{1}(\Omega)$, the nonlinear semigroup of global solutions associated to the problem (1.1)-(1.2) is shown to possess much of the properties of gradient systems (see [10]); namely,

Theorem 1.2. Assume that (1.4) holds, and that one of the conditions (H1), (H2) or (H3) in (1.7) is satisfied with $p=2$.

Then

i) for each $u_{0}, v_{0} \in H_{0}^{1}(\Omega)$ the solution $u\left(t, u_{0}, v_{0}\right)$ of (1.1) exists globally in time,

ii) relation $S(t)\left(\left[\begin{array}{l}u_{0} \\ v_{0}\end{array}\right]\right)=\left[\begin{array}{c}u\left(t, u_{0}, v_{0}\right) \\ u_{t}\left(t, u_{0}, v_{0}\right)\end{array}\right], t \geq 0, u_{0}, v_{0} \in H_{0}^{1}(\Omega)$, defines a $C^{0}$ semigroup on $H_{0}^{1}(\Omega) \times H_{0}^{1}(\Omega)$ with bounded orbits of bounded sets,

iii) the set $\mathcal{E}$ consisting of all the equilibria of $\{S(t)\}$ has the form $\mathcal{E}=\{(\phi, 0)$, $\left.\phi \in \mathcal{E}_{1}\right\}$, where $\mathcal{E}_{1}=\left\{\phi \in H_{0}^{1}(\Omega): A \phi=f(\phi)\right\}$ is a bounded subset of $C^{2}(\bar{\Omega})$,

iv) there is a continuous functional $\mathcal{V}: H_{0}^{1}(\Omega) \times H_{0}^{1}(\Omega) \rightarrow \mathbb{R}$ such that

a) $\mathcal{V}\left(S(\cdot)\left(\left[\begin{array}{l}u_{0} \\ v_{0}\end{array}\right]\right)\right)$ is bounded from below and nonincreasing in $[0, \infty)$ for any $\left[\begin{array}{l}u_{0} \\ v_{0}\end{array}\right] \in H_{0}^{1}(\Omega) \times H_{0}^{1}(\Omega)$,

b) $\left[\begin{array}{l}u_{0} \\ v_{0}\end{array}\right] \in H_{0}^{1}(\Omega) \times H_{0}^{1}(\Omega)$, and $\mathcal{V}\left(S(\cdot)\left(\left[\begin{array}{c}u_{0} \\ v_{0}\end{array}\right]\right)\right)=$ const. in $[0, \infty)$ implies that $\left[\begin{array}{l}u_{0} \\ v_{0}\end{array}\right] \in \mathcal{E}$,

v) for each $t \geq 0$ and $\left[\begin{array}{l}u_{0} \\ v_{0}\end{array}\right] \in W_{0}^{1, p}(\Omega) \times W_{0}^{1, p}(\Omega)$ the following formula holds:

$$
\begin{aligned}
& S(t)\left(\left[\begin{array}{l}
u_{0} \\
v_{0}
\end{array}\right]\right)=e^{-\mathcal{A}_{\mu} t}\left[\begin{array}{l}
u_{0} \\
v_{0}
\end{array}\right]+U\left(t,\left[\begin{array}{l}
u_{0} \\
v_{0}
\end{array}\right]\right), \\
& U\left(t,\left[\begin{array}{l}
u_{0} \\
v_{0}
\end{array}\right]\right):=\int_{0}^{t} e^{-\mathcal{A}_{\mu}(t-s)} \Phi_{1} \mathcal{F}\left(\Phi_{-1} S(s)\left(\left[\begin{array}{l}
u_{0} \\
v_{0}
\end{array}\right]\right)\right) d s,
\end{aligned}
$$

where

$$
e^{-\mathcal{A}_{\mu} t}=\sum_{k=0}^{\infty} \frac{(-1)^{k}}{k !} t^{k} \mathcal{A}_{\mu}^{k}
$$

\footnotetext{
${ }^{1}$ To simplify the notation we will next use for the extensions (resp. restrictions) of the operator $A$ the same symbol $A$.
} 
is the exponential function of the linear bounded operator $-\mathcal{A}_{\mu}$ given by (1.14) in $W_{0}^{1, p}(\Omega) \times W_{0}^{1, p}(\Omega)$ and $\Phi_{-1}: W_{0}^{1, p}(\Omega) \times W_{0}^{1, p}(\Omega) \rightarrow W_{0}^{-1, p}(\Omega) \times$ $W_{0}^{-1, p}(\Omega)$ is given by $\Phi_{-1}=\left[\begin{array}{cc}(I+A) & 0 \\ 0 & (I+A)\end{array}\right]$ and $\Phi_{1}=\left(\Phi_{-1}\right)^{-1}$.

As for the asymptotic properties of the solutions of (1.1)-(1.2) the following result is given.

Theorem 1.3. If (1.4) and (1.8) or (1.9) hold, then the semigroup $\{S(t)\}$ associated to the evolution problem (1.1) -(1.2) in $H_{0}^{1}(\Omega) \times H_{0}^{1}(\Omega)$ has a global attractor A given by (1.10).

When (1.8) holds, the global attractor $\mathbf{A}$ is bounded in $W^{2, q}(\Omega) \cap W_{0}^{1, q}(\Omega) \times$ $W^{2, q}(\Omega) \cap W_{0}^{1, q}(\Omega)$ for each $q \in(1, \infty)$ and thus compact in the space of Hölder functions $C^{1+\nu}(\bar{\Omega}) \times C^{1+\nu}(\bar{\Omega})$ for every $\nu \in(0,1)$.

Theorems 1.1 and 1.2 are proved in Section 2. In Section 3 the asymptotic regularity properties of $\{S(t)\}$ are obtained and the proof of Theorem 1.3 is completed under the assumption (1.8). In Section 4 we give the proof of (1.6) under the assumption (1.9) using Alekseev's nonlinear variation of constants formula (see 2], [5).

An open question remains whether the global attractor regularizes in the critical case (1.9), that is, when the critical exponent $\rho^{*}$ is involved.

\section{LOCAL AND GLOBAL WELL POSEDNESS RESUlts}

In what follows we will essentially use the properties of the double sided fractional power scale $\left\{E^{\alpha}, \alpha \in \mathbb{R}\right\}$ generated by $(E, \tilde{A})$, where $E$ is a reflexive Banach space and $\tilde{A}$ with the domain $E^{1}$ is a sectorial positive operator in $E$. In particular, we will use the properties of the scale corresponding to $\tilde{A}=-\Delta+I$ in $E=L^{p}(\Omega)=: E_{p}$ with the domain $E_{p}^{1}=W^{2, p}(\Omega) \cap W_{0}^{1, p}(\Omega)$, where $p \in(1, \infty)$ and $\Delta$ denotes the Dirichlet Laplacian.

We recall that the extrapolation space $E_{p}^{-\frac{1}{2}}$ of $E_{p}^{\frac{1}{2}}$ is the completion of $\left(E_{p}^{\frac{1}{2}},\left\|\left(\tilde{A}_{\frac{1}{2}}\right)^{-1} \cdot\right\|_{E_{p}^{\frac{1}{2}}}\right)$. We know that $E_{p}^{\frac{1}{2}}$ coincides with $W_{0}^{1, p}(\Omega)$ and from [1, Proposition V.1.4.3] we know that the (adjoint) space $\left(\left(E_{p^{\prime}}\right)^{\frac{1}{2}}\right)^{\prime}$ coincides with $E_{p}^{-\frac{1}{2}}$ and with the completion of $\left(E_{p},\left\|\tilde{A}^{-\frac{1}{2}} \cdot\right\|_{E_{p}}\right)$.

Following [1, Proposition V.1.4.3] we remark that we identify the elements $\phi \in$ $E_{p}^{-\frac{1}{2}}$ with the functionals from $\left(E_{p^{\prime}}^{\frac{1}{2}}\right)^{\prime}=\left(W_{0}^{1, p^{\prime}}(\Omega)\right)^{\prime}$ in such a way that the norm $\left\|\tilde{A}^{-\frac{1}{2}} \phi\right\|_{E_{p}}=:\|\phi\|_{E_{p}^{-\frac{1}{2}}}$ is equal to the norm $\|\phi\|_{L\left(E_{p^{\prime}}^{\frac{1}{2}}, \mathbb{C}\right)}=\|\phi\|_{\left(W_{0}^{1, p^{\prime}}(\Omega)\right)^{\prime}}=$ $\|\phi\|_{W^{-1, p}(\Omega)}$ whenever $\phi \in E_{p}$. For $\phi \in E_{p}^{-\frac{1}{2}} \backslash E_{p}$ we then have $\|\phi\|_{L\left(E_{p^{\prime}}^{\frac{1}{2}}, \mathbb{C}\right)}=$ $\|\phi\|_{\left(W_{0}^{1, p^{\prime}}(\Omega)\right)^{\prime}}=\|\phi\|_{W^{-1, p}(\Omega)}=\lim _{j \rightarrow \infty}\left\|\tilde{A}^{-\frac{1}{2}} \phi_{j}\right\|_{E_{p}}$ for any suitable (Cauchy) sequence $\left\{\phi_{j}\right\} \subset E$.

As for the representation of the functionals we obtain the following relation.

Lemma 2.1. If $E_{2}=L^{2}(\Omega)$ and $\tilde{A}$ is the negative Dirichlet Laplacian plus any multiple of the identity such that $\tilde{A}$ is positive with the domain $E_{2}^{1}=H^{2}(\Omega) \cap H_{0}^{1}(\Omega)$, then

$$
\left\langle\tilde{A}^{-\frac{1}{2}} \phi, \tilde{A}^{\frac{1}{2}} \psi\right\rangle_{E_{2}}=\int_{\Omega} \phi \psi d s, \quad \phi \in E_{\frac{2 N}{N+2}}, \psi \in E_{2}^{\frac{1}{2}}
$$


Proof. If $E_{2}^{\frac{1}{2}} \ni \phi_{n} \stackrel{E_{\frac{2 N}{N+2}}^{\rightarrow}}{\rightarrow} \phi \in E_{\frac{2 N}{N+2}}$, then $\phi_{n} \stackrel{E_{2}^{-\frac{1}{2}}}{\rightarrow} \phi$ and for $\psi \in E_{2}^{\frac{1}{2}}$ we get

$$
\left\langle\tilde{A}^{-\frac{1}{2}} \phi, \tilde{A}^{\frac{1}{2}} \psi\right\rangle_{E_{2}} \leftarrow\left\langle\tilde{A}^{-\frac{1}{2}} \phi_{n}, \tilde{A}^{\frac{1}{2}} \psi\right\rangle_{E_{2}}=\int_{\Omega} \phi_{n} \psi d x \rightarrow \int_{\Omega} \phi \psi d s
$$

which proves the result.

As for the properties of $\mathcal{A}_{\mu}$ defined by (1.14) in $E_{p}^{-\frac{1}{2}} \times E_{p}^{-\frac{1}{2}}$ we prove the following lemma.

Lemma 2.2. Let $p \in(1, \infty)$ and $\mu>0$. Suppose that $A$ is the negative Dirichlet Laplacian in $L^{p}(\Omega)$ with the domain $W^{2, p}(\Omega) \cap W_{0}^{1, p}(\Omega)$ and consider its closed extension to $\left(W_{0}^{1, p^{\prime}}(\Omega)\right)^{\prime}=: W^{-1, p}(\Omega)$. Then $\mathcal{A}_{\mu}$ is a bounded linear operator in $E_{p}^{-\frac{1}{2}} \times E_{p}^{-\frac{1}{2}}$ and the uniformly continuous semigroup $e^{-\mathcal{A}_{\mu} t}=\sum_{k=0}^{\infty} \frac{(-1)^{k}}{k !} t^{k} \mathcal{A}_{\mu}^{k}$ generated by $-\mathcal{A}_{\mu}$ in $E_{p}^{-\frac{1}{2}} \times E_{p}^{-\frac{1}{2}}$ satisfies the estimate

$$
\left\|e^{-\mathcal{A}_{\mu} t}\right\|_{L\left(E_{p}^{-\frac{1}{2}} \times E_{p}^{-\frac{1}{2}}\right)} \leq M_{p} e^{-\omega t}, t \geq 0
$$

with certain positive constants $M_{p}, \omega$.

Proof. If we write the equation $\left(\lambda I+\mathcal{A}_{\mu}\right)\left[\begin{array}{l}\phi_{1} \\ \phi_{2}\end{array}\right]=\left[\begin{array}{l}\psi_{1} \\ \psi_{2}\end{array}\right]$ we will observe that

$$
\left[\begin{array}{l}
\phi_{1} \\
\phi_{2}
\end{array}\right]=\left[\begin{array}{c}
\left(\left(\lambda^{2}+\mu \lambda+1\right) I-(\mu \lambda+1)(I+A)^{-1}\right)^{-1}\left(\lambda \psi_{1}+\mu\left(I-(I+A)^{-1}\right) \psi_{1}+\psi_{2}\right) \\
\lambda\left(\left(\lambda^{2}+\mu \lambda+1\right) I-(\mu \lambda+1)(I+A)^{-1}\right)^{-1}\left(\lambda \psi_{1}+\mu\left(I-(I+A)^{-1}\right) \psi_{1}+\psi_{2}\right)-\psi_{1}
\end{array}\right]
$$

whenever the inverse operator $\left(\left(\lambda^{2}+\mu \lambda+1\right) I-(\mu \lambda+1)(I+A)^{-1}\right)^{-1}$ is well defined. From the spectral mapping theorem (see [7, p. 243]) we know that

$$
\sigma\left((I+A)^{-1}\right)-\{0\}=\left\{(1+\nu)^{-1}, \nu \in \sigma(A)\right\},
$$

which shows that $\sigma\left((I+A)^{-1}\right) \subset\left[0,\left(1+\lambda_{1}\right)^{-1}\right]$.

Since, for $\lambda \neq-\mu^{-1}$,

$$
\operatorname{Im}\left(\lambda^{2}(1+\mu \lambda)^{-1}+1\right)=\frac{\mu \operatorname{Im} \lambda}{(1+\mu \operatorname{Re} \lambda)^{2}+(\mu \operatorname{Im} \lambda)^{2}}\left[(\operatorname{Im} \lambda)^{2}+\left(\operatorname{Re} \lambda+\frac{1}{\mu}\right)^{2}-\frac{1}{\mu^{2}}\right]
$$

we have that:

- the points in $\sigma\left(-\mathcal{A}_{\mu}\right)$ with $\operatorname{Im} \lambda \neq 0$ must be of the form $\lambda=-\frac{1}{\mu}+\frac{1}{\mu} e^{i \theta}$. For $\lambda=-\frac{1}{\mu}+\frac{1}{\mu} e^{i \theta}$ we have that $\lambda^{2}(1+\mu \lambda)^{-1}+1=2 \mu^{-2}(\cos \theta-1)+1$. Now, for $\mu^{2}>4 \lambda_{1}^{-1}\left(1+\lambda_{1}\right)$ and $\theta \in \mathbb{R}$ or for $\mu^{2} \leq 4 \lambda_{1}^{-1}\left(1+\lambda_{1}\right)$ and $0<|\theta|<\theta_{0}:=\arccos \left(\frac{2+\left(2-\mu^{2}\right) \lambda_{1}}{2+2 \lambda_{1}}\right)$, we have that $\lambda=-\mu^{-1}+\mu^{-1} e^{i \theta} \in$ $\rho\left(-\mathcal{A}_{\mu}\right)$. This shows that $\lambda \in \rho\left(-\mathcal{A}_{\mu}\right)$ whenever $\operatorname{Re} \lambda>-\frac{\mu \lambda_{1}}{2+2 \lambda_{1}}, \operatorname{Im} \lambda \neq 0$;

- the points with $\operatorname{Im} \lambda=0$ which satisfy the condition $\lambda^{2}(\mu \lambda+1)^{-1}+1>$ $\left(1+\lambda_{1}\right)^{-1}$ are in $\rho\left(-\mathcal{A}_{\mu}\right)$. Hence, we clearly have

$$
\rho\left(-\mathcal{A}_{\mu}\right) \cap \mathbb{R} \supset\left(-\mu^{-1}, \infty\right) .
$$

Thus, $\rho\left(-\mathcal{A}_{\mu}\right) \supset\left\{\lambda \in \mathbb{C}: \operatorname{Re} \lambda>-\min \left\{\mu^{-1}, \mu \lambda_{1}\left(2+2 \lambda_{1}\right)^{-1}\right\}\right\}$ and (2.2) is proved.

As for the isometric properties of the operator $\tilde{A}$ we specifically mention the following result (see [1, Corollary 1.3.9]). 
Lemma 2.3. If $p \in(1, \infty), s \in[0, \infty)$ and $\Phi_{s}: E_{p}^{-\frac{1}{2}} \times E_{p}^{-\frac{1}{2}} \rightarrow E_{p}^{s-\frac{1}{2}} \times E_{p}^{s-\frac{1}{2}}$ is given in the matrix form as

$$
\Phi_{s}=\left[\begin{array}{cc}
(I+A)^{-s} & 0 \\
0 & (I+A)^{-s}
\end{array}\right]
$$

then $\Phi_{s}$ is an isometric isomorphism 2 from $E_{p}^{r} \times E_{p}^{r}$ onto $E_{p}^{r+s} \times E_{p}^{r+s}$ for each $r \geq-\frac{1}{2}$; in particular $\Phi_{1}$ is an isometric isomorphism from $E_{p}^{-\frac{1}{2}} \times E_{p}^{-\frac{1}{2}}$ onto $E_{p}^{\frac{1}{2}} \times E_{p}^{\frac{1}{2}}$.

2.1. Local well posedness result and the proof of Theorem 1.1. We first prove the auxiliary lemma below.

Lemma 2.4. Let $p \in(1, \infty), f: \mathbb{R} \rightarrow \mathbb{R}$, and $A$ be as in Lemma 2.2. Then

$$
f^{e}(\phi)(x)=f\left((I+A)^{-1} \phi(x)\right), x \in \Omega,
$$

defines an operator from $W^{-1, p}(\Omega)$ into $W^{-1, p}(\Omega)$ which is Lipschitz continuous in bounded sets provided that one of the conditions (H1), (H2) or (H3) in (1.7) holds.

Proof. Let $B$ be bounded in $E_{p}^{-\frac{1}{2}}$ and choose arbitrary $\phi_{1}, \phi_{2} \in B$.

If condition (H1) is satisfied, then $(I+A)^{-1} B$ is a bounded subset of $E_{p}^{\frac{1}{2}}$ and hence of $C^{0}(\bar{\Omega})$. Since $f$ is Lipschitz continuous on bounded subsets of $\mathbb{R}^{1}$ we have

$$
\begin{aligned}
& \left\|f^{e}\left(\phi_{1}\right)-f^{e}\left(\phi_{2}\right)\right\|_{E_{p}^{-\frac{1}{2}}} \leq C\left\|f^{e}\left(\phi_{1}\right)-f^{e}\left(\phi_{2}\right)\right\|_{E_{p}^{0}} \\
& \leq C L\left\|(I+A)^{-1}\left(\phi_{1}-\phi_{2}\right)\right\|_{E_{p}^{0}} \leq \tilde{C} L\left\|\phi_{1}-\phi_{2}\right\|_{E_{p}^{-\frac{1}{2}}} .
\end{aligned}
$$

When either (H2) or (H3) holds we use the Sobolev and Hölder inequalities to get

$$
\begin{aligned}
& \left\|f^{e}\left(\phi_{1}\right)-f^{e}\left(\phi_{2}\right)\right\|_{E_{p}^{-\frac{1}{2}}} \leq C\left\|f^{e}\left(\phi_{1}\right)-f^{e}\left(\phi_{2}\right)\right\|_{E_{\frac{N p}{N+p}}} \\
& =C\left\|f\left((I+A)^{-1} \phi_{1}\right)-f\left((I+A)^{-1} \phi_{2}\right)\right\|_{E_{\frac{N p}{N+p}}} \\
& \leq \hat{C}\left\|(I+A)^{-1}\left(\phi_{1}-\phi_{2}\right)\right\|_{E_{p}^{\frac{1}{2}}}\left(1+\left\|(I+A)^{-1} \phi_{1}\right\|_{E_{\frac{N(\rho-1)}{2}}^{\rho-1}}+\left\|(I+A)^{-1} \phi_{2}\right\|_{E_{\frac{N(\rho-1)}{2}}^{\rho-1}}^{2}\right) \\
& \leq \tilde{C}\left\|(I+A)^{-1}\left(\phi_{1}-\phi_{2}\right)\right\|_{E_{p}^{\frac{1}{2}}}\left(1+\left\|(I+A)^{-1} \phi_{1}\right\|_{E_{p}^{\frac{1}{2}}}^{\rho-1}+\left\|(I+A)^{-1} \phi_{2}\right\|_{E_{p}^{\rho-1}}^{\rho-1}\right) \\
& \leq \tilde{C}\left\|\phi_{1}-\phi_{2}\right\|_{E_{p}^{-\frac{1}{2}}}\left(1+\left\|\phi_{1}\right\|_{E_{p}^{-\frac{1}{2}}}^{\rho-1}+\left\|\phi_{2}\right\|_{E_{p}^{-\frac{1}{2}}}^{\rho-1}\right){ }_{E_{p}^{-\frac{1}{2}}} .
\end{aligned}
$$

The local well posedness result for (1.13) in $W^{-1, p}(\Omega) \times W^{-1, p}(\Omega)$ now follows.

Theorem 2.1. If the assumptions of Lemma 2.4 are satisfied and one of the conditions (H1), (H2) or (H3) in (1.7) holds, then for each $v_{0}, w_{0} \in W^{-1, p}(\Omega)$ the problem (1.13) has a unique solution $\left[\begin{array}{c}v \\ w\end{array}\right]\left(\cdot, v_{0}, w_{0}\right) \in C\left(\left[0, \tau_{v_{0}, w_{0}}\right), W^{-1, p}(\Omega) \times W^{-1, p}(\Omega)\right)$ defined on the maximal interval of existence $\left[0, \tau_{v_{0}, w_{0}}\right)$ and continuously depending

\footnotetext{
${ }^{2}$ Recall that we consider the double sided fractional power scale $\left\{E^{\alpha}, \alpha \in \mathbb{R}\right\}$ generated by $(E, \tilde{A})$ with $\tilde{A}=I+A$; in particular for $\phi \in E_{p}^{\frac{1}{2}}$ we have that $\|\phi\|_{E_{p}^{\frac{1}{2}}}=\left\|(I+A)^{\frac{1}{2}} \phi\right\|_{E_{p}}$ and $\|\phi\|_{E_{p}^{-\frac{1}{2}}}=\left\|(I+A)^{-\frac{1}{2}} \phi\right\|_{E_{p}}$.
} 
on the initial data, where either $\tau_{v_{0}, w_{0}}=\infty$ or $\left\|v\left(t, v_{0}, w_{0}\right)\right\|_{X}+\left\|w\left(t, v_{0}, w_{0}\right)\right\|_{X} \rightarrow$ $\infty$ as $t \rightarrow \tau_{v_{0}, w_{0}}^{-}$. The solution satisfies in $W^{-1, p}(\Omega)$ the variation of constants formula

$$
\left[\begin{array}{l}
v \\
w
\end{array}\right]\left(\cdot, v_{0}, w_{0}\right)=e^{-\mathcal{A}_{\mu} t}\left[\begin{array}{c}
v_{0} \\
w_{0}
\end{array}\right]+\int_{0}^{t} e^{-\mathcal{A}_{\mu}(t-s)}\left[\begin{array}{c}
0 \\
f^{e}\left(v\left(s, v_{0}, w_{0}\right)\right)
\end{array}\right] d s, t \in\left[0, \tau_{v_{0}, w_{0}}\right),
$$

with $\left\{e^{-\mathcal{A}_{\mu} t}\right\}$ defined in Lemma 2.2 and, in addition,

$$
\begin{aligned}
& {\left[\begin{array}{c}
v \\
w
\end{array}\right]\left(\cdot, v_{0}, w_{0}\right) \in C^{1}\left(\left[0, \tau_{v_{0}, w_{0}}\right), W^{-1, p}(\Omega) \times W^{-1, p}(\Omega)\right),} \\
& {\left[\begin{array}{c}
v_{t} \\
w_{t}
\end{array}\right]\left(\cdot, v_{0}, w_{0}\right) \in C^{1}\left(\left[0, \tau_{v_{0}, w_{0}}\right), W^{-1, p}(\Omega) \times W^{-1, p}(\Omega)\right) .}
\end{aligned}
$$

In particular, $\left[\begin{array}{c}v \\ w\end{array}\right]\left(\cdot, v_{0}, w_{0}\right)$ is a classical solution of (1.13) in $X \times X:=W^{-1, p}(\Omega) \times$ $W^{-1, p}(\Omega)$.

Proof. Let $p \in(1, \infty)$ and $X=E_{p}^{-\frac{1}{2}}=W^{-1, p}(\Omega)$. From Lemma 2.4, if $B$ is a bounded subset of $X \times X$ and $\left[\begin{array}{l}\phi_{1} \\ \psi_{1}\end{array}\right],\left[\begin{array}{l}\phi_{2} \\ \psi_{2}\end{array}\right] \in B$, we obtain

$$
\left\|\mathcal{F}\left(\left[\begin{array}{l}
\phi_{1} \\
\psi_{1}
\end{array}\right]\right)-\mathcal{F}\left(\left[\begin{array}{l}
\phi_{2} \\
\psi_{2}
\end{array}\right]\right)\right\|_{X \times X}=\left\|f^{e}\left(\phi_{1}\right)-f^{e}\left(\phi_{2}\right)\right\|_{E_{p}^{-\frac{1}{2}}} \leq c\left\|\left[\begin{array}{l}
\phi_{1} \\
\psi_{1}
\end{array}\right]-\left[\begin{array}{l}
\phi_{2} \\
\psi_{2}
\end{array}\right]\right\|_{X \times X} .
$$

Hence, $\mathcal{F}$ takes $X \times X$ into $X \times X$ and is Lipschitz continuous on bounded sets so that the semigroup theory applies (see [12, 15), from which the results follow easily.

The proof of Theorem 1.1. The local well posedness of (1.1) in $W_{0}^{1, p}(\Omega) \times$ $W_{0}^{1, p}(\Omega)$ follows from Theorem 2.1 and Lemma 2.3. For this we define $\tau\left(u_{0}, v_{0}\right):=$ $\tau_{(I+A) u_{0},(I+A) v_{0}}$ and

$$
u(t)=(I+A)^{-1} v(t), u_{t}(t)=(I+A)^{-1} v_{t}(t),
$$

where $\left[\begin{array}{c}v \\ v_{t}\end{array}\right]$ is the (uniquely defined on $\left[0, \tau\left(u_{0}, v_{0}\right)\right.$ ) solution of (1.13) from Theorem 2.1 .

2.2. Global solutions to (1.1) and the proof of Theorem 1.2, Assuming (1.4) we obtain below the global well posedness result for (1.1)-(1.2) and prove Theorem 1.2

Lemma 2.5. Under the assumptions and notation of Theorem 1.1 condition (1.4) implies the existence of a constant $C>0$ such that if $u_{0}, v_{0} \in W_{0}^{1, p}(\Omega)$ and $p \in$ $[2, \infty)$, then the functions $u\left(t, u_{0}, v_{0}\right)$ and $u_{t}\left(t, u_{0}, v_{0}\right)$ fulfill the estimate

$$
\left\|u_{t}\right\|_{E_{2}^{\frac{1}{2}}}^{2}+\|u\|_{E_{2}^{\frac{1}{2}}}^{2} \leq C\left(1+\left\|u_{0}\right\|_{E_{2}^{\frac{1}{2}}}^{\rho+1}+\left\|v_{0}\right\|_{E_{2}^{\frac{1}{2}}}^{2}\right), t \in\left[0, \tau\left(u_{0}, v_{0}\right)\right) .
$$

Proof. From Theorem 2.1 function $v=(I+A) u$ solves (1.11) in $E_{2}^{-\frac{1}{2}}$. We thus take the $E_{2}^{-\frac{1}{2}}$ product $3\langle\cdot, \cdot\rangle_{E_{2}^{-\frac{1}{2}}}$ of each side of (1.11) with $v_{t}$ to get

$$
\begin{aligned}
& \frac{d}{d t} \frac{1}{2}\left(\left\|v_{t}\right\|_{E_{2}^{-\frac{1}{2}}}^{2}+\|v\|_{E_{2}^{-\frac{1}{2}}}^{2}-\left\|(I+A)^{-\frac{1}{2}} v\right\|_{E_{2}^{-\frac{1}{2}}}^{2}\right)-\left\langle f^{e}(v), v_{t}\right\rangle_{E_{2}^{-\frac{1}{2}}} \\
& =-\mu\left(\left\|v_{t}\right\|_{E_{2}^{-\frac{1}{2}}}-\left\|(I+A)^{-\frac{1}{2}} v_{t}\right\|_{E_{2}^{-\frac{1}{2}}}\right) .
\end{aligned}
$$

\footnotetext{
${ }^{3}$ Since $\left\{E^{\alpha}, \alpha \in \mathbb{R}\right\}$ is generated by $(E, \tilde{A})$ with $\tilde{A}=I+A$, then the product $\langle\phi, \psi\rangle_{E_{2}}{ }^{-\frac{1}{2}}$ of $\phi, \psi \in E_{2}^{-\frac{1}{2}}$ is given by $\left\langle(I+A)^{-\frac{1}{2}} \phi,(I+A)^{-\frac{1}{2}} \psi\right\rangle_{E_{2}},\langle\cdot, \cdot\rangle_{E_{2}}$ being the (original) scalar product in $E_{2}$.
} 
The Poincaré inequality reads

$$
\left\|(I+A)^{\frac{1}{2}} \phi\right\|_{E_{2}}^{2} \geq\left(1+\lambda_{1}\right)\|\phi\|_{E_{2}}^{2}, \phi \in E_{2}^{\frac{1}{2}},
$$

which for $\phi=(I+A)^{-1} \psi$ translates into the estimates

$$
\|\psi\|_{E_{2}^{-\frac{1}{2}}}^{2} \geq\left(1+\lambda_{1}\right)\left\|(I+A)^{-\frac{1}{2}} \psi\right\|_{E_{2}^{-\frac{1}{2}}}^{2}, \psi \in E_{2}^{-\frac{1}{2}},
$$

and implies

$$
\begin{aligned}
& \frac{\lambda_{1}}{1+\lambda_{1}}\|v\|_{E_{2}^{-\frac{1}{2}}}^{2} \leq\|v\|_{E_{2}^{-\frac{1}{2}}}^{2}-\left\|(I+A)^{-\frac{1}{2}} v\right\|_{E_{2}^{-\frac{1}{2}}}^{2}, \\
& \frac{\lambda_{1}}{1+\lambda_{1}}\left\|v_{t}\right\|_{E_{2}^{-\frac{1}{2}}}^{2} \leq\left\|v_{t}\right\|_{E_{2}^{-\frac{1}{2}}}^{2}-\left\|(I+A)^{-\frac{1}{2}} v_{t}\right\|_{E_{2}^{-\frac{1}{2}}}^{2} .
\end{aligned}
$$

From Lemma 2.1 we get

$$
\left\langle(I+A)^{-\frac{1}{2}} f^{e}(v),(I+A)^{\frac{1}{2}}(I+A)^{-1} v_{t}\right\rangle_{E_{2}}=\int_{\Omega} f^{e}(v)(I+A)^{-1} v_{t} d x,
$$

and hence

$$
\begin{aligned}
\left\langle f^{e}(v), v_{t}\right\rangle_{E_{2}^{-\frac{1}{2}}} & =\left\langle(I+A)^{-\frac{1}{2}} f^{e}(v),(I+A)^{-\frac{1}{2}} v_{t}\right\rangle_{E_{2}} \\
& =\int_{\Omega} f\left((I+A)^{-1} v\right)(I+A)^{-1} v_{t} d x
\end{aligned}
$$

If $F$ is the primitive function of $f$ in $\mathbb{R}^{1}$ we then have

$$
\int_{\Omega} f\left((I+A)^{-1} v\right)(I+A)^{-1} v_{t} d x=\frac{d}{d t} \int_{\Omega} F\left((I+A)^{-1} v\right) d x .
$$

We now remark that (1.4) implies the existence of constants $\epsilon, \mathcal{C}>0$, for which

$$
F(t)=\int_{0}^{t} f(s) d s \leq \frac{1}{2}\left(\lambda_{1}-\epsilon\right) t^{2}+\mathcal{C}, t \in \mathbb{R}^{1} .
$$

As a consequence we infer

$$
\left.\left\langle F\left((I+A)^{-1} v\right), 1\right\rangle_{E_{2}} \leq \frac{1}{2}\left(\lambda_{1}-\epsilon\right) \|(I+A)^{-\frac{1}{2}} v\right) \|_{E_{2}^{-\frac{1}{2}}}^{2}+\mathcal{C}|\Omega|,
$$

which with the aid of (2.10) reads

$$
\int_{\Omega} F\left((I+A)^{-1} v\right) d x \leq \frac{\lambda_{1}-\epsilon}{2\left(1+\lambda_{1}\right)}\|v\|_{E_{2}^{-\frac{1}{2}}}^{2}+\mathcal{C}|\Omega| .
$$

Connecting (2.8)-(2.13) we get for

$$
\mathcal{L}\left(v, v_{t}\right):=\frac{1}{2}\left\|v_{t}\right\|_{E_{2}^{-\frac{1}{2}}}^{2}+\frac{1}{2}\|v\|_{E_{2}^{-\frac{1}{2}}}^{2}-\frac{1}{2}\left\|(I+A)^{-\frac{1}{2}} v\right\|_{E_{2}^{-\frac{1}{2}}}^{2}-\int_{\Omega} F\left((I+A)^{-1} v\right) d x
$$

that

$$
\frac{d}{d t}\left(\mathcal{L}\left(v, v_{t}\right)\right)=-\mu\left(\left\|v_{t}\right\|_{E_{2}^{-\frac{1}{2}}}-\left\|(I+A)^{-\frac{1}{2}} v_{t}\right\|_{E_{2}^{-\frac{1}{2}}}\right) \leq-\frac{\mu \lambda_{1}}{1+\lambda_{1}}\left\|v_{t}\right\|_{E_{2}^{-\frac{1}{2}}}^{2} \leq 0
$$


and hence

$$
\frac{1}{2}\left\|v_{t}\right\|_{E_{2}^{-\frac{1}{2}}}^{2}+\frac{\epsilon}{2\left(1+\lambda_{1}\right)}\|v\|_{E_{2}^{-\frac{1}{2}}}^{2}-\mathcal{C}|\Omega| \leq \mathcal{L}\left(v, v_{t}\right) \leq \mathcal{L}\left(v(0), v_{t}(0)\right),
$$

as long as the solution $v$ exists.

In the variables $(t, u)$ we then have

$\left\|u_{t}\right\|_{E_{2}^{\frac{1}{2}}}^{2}+\frac{\epsilon}{1+\lambda_{1}}\|u\|_{E_{2}^{\frac{1}{2}}}^{2} \leq\left\|v_{0}\right\|_{E_{2}^{\frac{1}{2}}}^{2}+\left\|(I+A)^{\frac{1}{2}} u_{0}\right\|_{E_{2}}^{2}-\left\|u_{0}\right\|_{E_{2}}^{2}-2 \int_{\Omega} F\left(u_{0}\right) d x+2 \mathcal{C}|\Omega|$,

where

$$
\left\|F\left(u_{0}\right)\right\|_{L^{1}(\Omega)} \leq \text { const. }\left(1+\left\|u_{0}\right\|_{E_{2}^{\frac{1}{2}}}^{\rho+1}\right),
$$

since (1.7) implies that $|F(s)| \leq$ const. $\left(1+|s|^{\rho+1}\right)$ for $s \in \mathbb{R}$. Thus (2.7) follows from (2.16) and (2.17) via the Poincaré inequality (2.9).

Proof of Theorem 1.2. Parts i) and ii) follow from Lemma 2.5 and Theorem 1.1

To prove the boundedness of $\mathcal{E}_{1}$ in $H_{0}^{1}(\Omega)$ we first infer from Lemma 2.1 that

$$
\left\langle A^{-\frac{1}{2}} f(u), A^{\frac{1}{2}} u\right\rangle_{E_{2}}=\int_{\Omega} f(u) u d x .
$$

Since $u \in \mathcal{E}_{1}$ implies that $u \in E_{2}^{\frac{1}{2}}$ and $A^{\frac{1}{2}} u=A^{-\frac{1}{2}} f(u)$, then

$$
\left\langle A^{\frac{1}{2}} u, A^{\frac{1}{2}} u\right\rangle_{E_{2}}=\int_{\Omega} f(u) u d x, u \in \mathcal{E}_{1} .
$$

With the aid of (1.4) and the Poincaré inequality we then get

$$
\left\|A^{\frac{1}{2}} u\right\|_{E_{2}}^{2} \leq\left(\lambda_{1}-\epsilon\right)\|u\|_{E_{2}}^{2}+C|\Omega| \leq\left(1-\epsilon \lambda_{1}^{-1}\right)\left\|A^{\frac{1}{2}} u\right\|_{E_{2}}^{2}+C|\Omega|
$$

for some $\epsilon, C>0$ and hence

$$
\sup _{u \in \mathcal{E}_{1}}\left\|A^{\frac{1}{2}} u\right\|_{E_{2}}^{2} \leq C|\Omega| \lambda_{1} \epsilon^{-1}=: M_{1} .
$$

Referring next to [3, Theorem 2.6], we infer that $\mathcal{E}_{1}$ is bounded in $L^{\infty}(\Omega)$ and hence in $W^{2, q}(\Omega) \cap W_{0}^{1, q}(\Omega)$ for each $q \in(1, \infty)$. In particular, for any equilibrium solution $\left[\begin{array}{l}\phi \\ 0\end{array}\right]$ the map $f(\phi)$ is of class $C^{1+\nu}(\bar{\Omega})$ for each $\nu \in(0,1)$. Therefore $\phi \in C^{2+\nu}(\bar{\Omega})$ and $\mathcal{E}_{1}$ is bounded in $C^{2+\nu}(\bar{\Omega})$ as a consequence of the Schauder type estimates (see [9, Theorems 9.19,6.6]).

For the proof of part iv) we come back to (2.14), defining

$\mathcal{V}\left(S(t)\left(\left[\begin{array}{c}u_{0} \\ v_{0}\end{array}\right]\right)\right):=\mathcal{L}\left(\Phi_{-1} S(t)\left(\left[\begin{array}{c}u_{0} \\ v_{0}\end{array}\right]\right)\right)=\frac{1}{2}\left\|u_{t}\right\|_{E_{2}^{\frac{1}{2}}}^{2}+\frac{1}{2}\|u\|_{E_{2}^{\frac{1}{2}}}^{2}-\frac{1}{2}\|u\|_{E_{2}}^{2}-\int_{\Omega} F(u) d x$.

Following the assumptions on $f$ for $\phi, \tilde{\phi} \in E_{2}^{\frac{1}{2}}$ we have

$$
\begin{aligned}
\|F(\phi)-F(\tilde{\phi})\|_{L^{1}(\Omega)} & \leq \tilde{c}\left\||\phi-\tilde{\phi}|\left(1+|\phi|^{\rho}+|\tilde{\phi}|^{\rho}\right)\right\|_{L^{1}(\Omega)} \\
& \leq \hat{c}\|\phi-\tilde{\phi}\|_{E_{2}^{\frac{1}{2}}}\left(1+\|\phi\|_{E_{2}^{\frac{1}{2}}}^{\rho}+\|\tilde{\phi}\|_{E_{2}^{\frac{1}{2}}}^{\rho} .\right.
\end{aligned}
$$

Properties a) and b) follow then from Lemma 2.5. 
For the proof of v) we combine (1.15), (2.4) and (2.6) to get

$$
\begin{array}{r}
{\left[\begin{array}{c}
(I+A) u \\
(I+A) u_{t}
\end{array}\right](t)=e^{-\mathcal{A}_{\mu} t}\left[\begin{array}{c}
(I+A) u_{0} \\
(I+A) v_{0}
\end{array}\right]+\int_{0}^{t} e^{-\mathcal{A}_{\mu}(t-s)} \mathcal{F}\left(\left[\begin{array}{c}
(I+A) u(s) \\
(I+A) u_{t}(s)
\end{array}\right]\right) d s,} \\
t \geq 0, \quad\left[\begin{array}{c}
u_{0} \\
v_{0}
\end{array}\right] \in E_{2}^{\frac{1}{2}} .
\end{array}
$$

We also know that $\Phi_{1} \in L\left(E_{2}^{-\frac{1}{2}} \times E_{2}^{-\frac{1}{2}}\right)$ and $\mathcal{A}_{\mu}$ commutes with $\Phi_{1}$. Therefore, $e^{-\mathcal{A}_{\mu} t}$ commutes with $\Phi_{1}$ and an application of $\Phi_{1}$ to both sides of (2.23) gives the result.

\section{Asymptotic REgUlarity PROPERTIES of $\{S(t)\}$ AND REGUlarity}

OF THE GLOBAL ATTRACTOR IN THE SUBCRITICAL CASE

In this section we prove the asymptotic regularity properties of $\{S(t)\}$ assuming condition (1.8). We then obtain the existence and regularity properties of the global attractor proving Theorem 1.3 under assumption (1.8). The existence of an attractor for the case (1.9) will be proved in Section 4 .

Before we proceed with the proof of Theorem 1.3 we need the following two lemmas.

Lemma 3.1. If condition (1.8) holds, then $\tilde{f}^{e}=f^{e} \circ(I+A)$ takes sets bounded in $E_{2}^{\frac{1}{2}}$ into sets bounded in $E_{\frac{2 N}{N+2(1-\tilde{\sigma})}}$ and thus also in $E_{2}^{\frac{\tilde{\sigma}-1}{2}}$ with $\tilde{\sigma}=\min \left\{1, \frac{N+2}{2}-\right.$ $\left.\rho \frac{N-2}{2}\right\}$. In particular, if $N=1$ or $N=2$, then $\tilde{f}^{e}$ takes bounded subsets of $E_{2}^{\frac{1}{2}}$ into bounded subsets of $E_{q}$ for every $q \in(1, \infty)$.

Proof. Let $N \geq 3$ and recall that $E_{2}^{\frac{1-\sigma}{2}} \hookrightarrow E_{\frac{2 N}{N-2(1-\sigma)}}$ for $\sigma \in[0,1]$. Recall also that $E_{2}^{\frac{\sigma-1}{2}}=\left(E_{2}^{\frac{1-\sigma}{2}}\right)^{\prime}$. If $1<\rho<\frac{N+2}{N-2}$, then (1.7) leads for $\phi \in E_{2}^{\frac{1}{2}}$ to the condition

$$
\left\|f^{e}((I+A) \phi)\right\|_{E_{2} \frac{\tilde{\sigma}-1}{2}} \leq \tilde{c}\|f(\phi)\|_{E_{N+2(1-\tilde{\sigma})}} \leq \bar{c}\left\|1+|\phi|^{\rho}\right\|_{E_{\bar{N}+2(1-\tilde{\sigma})}} \leq \hat{c}\left(1+\|\phi\|_{E_{2}^{\frac{1}{2}}}^{\rho}\right) .
$$

If $N=1$ or $N=2$, then

$$
\|f(\phi)\|_{E_{q}} \leq \bar{c}\left\|1+|\phi|^{\rho}\right\|_{E_{q}} \leq \bar{c}\left(\|1\|_{E_{q}}+\|\phi\|_{E_{\rho q}}^{\rho}\right) \leq \hat{c}\left(1+\|\phi\|_{E_{2}^{\frac{1}{2}}}^{\rho}\right), \phi \in E_{2}^{\frac{1}{2}},
$$

since in this case $E_{2}^{\frac{1}{2}} \hookrightarrow E_{r}$ with any $r \in(1, \infty)$.

Lemma 3.2. Suppose that $p \in(1, \infty)$, that $A$ is as in Lemma 2.2 and that (1.4) and (1.8) hold. Then, for any set $B$ bounded in $E_{2}^{\frac{1}{2}} \times E_{2}^{\frac{1}{2}}$ and $M_{p}, \omega, \hat{c}$ as in (2.2), (3.1) we have

( $\alpha$ ) in the case $N \geq 3$,

$$
\begin{aligned}
& \sup _{\left[\begin{array}{c}
u_{0} \\
v_{0}
\end{array}\right] \in B} \sup _{t \geq 0}\left\|\int_{0}^{t} e^{-\mathcal{A}_{\mu}(t-s)} \Phi_{1} \mathcal{F}\left(\Phi_{-1} S(s)\left(\left[\begin{array}{l}
u_{0} \\
v_{0}
\end{array}\right]\right)\right) d s\right\|_{E_{2}^{\frac{1+\tilde{\sigma}}{2}} \times E_{2}^{\frac{1+\tilde{\sigma}}{2}}} \\
& \qquad \frac{M_{2} \hat{c}}{\omega}\left(1+\sup _{\left[\begin{array}{c}
u_{0} \\
v_{0}
\end{array}\right] \in \gamma^{+}(B)}\left\|u_{0}\right\|_{E_{2}^{\frac{1}{2}}}^{\rho}\right) \\
& \text { with } \tilde{\sigma}:=\min \left\{1, \frac{N}{2}+1-\rho\left(\frac{N}{2}-1\right)\right\}
\end{aligned}
$$


and

( $\beta$ ) in the case $N=1$ or $N=2$,

$$
\begin{gathered}
\sup _{\left[\begin{array}{l}
u_{0} \\
v_{0}
\end{array}\right] \in B} \sup _{t \geq 0}\left\|\int_{0}^{t} e^{-\mathcal{A}_{\mu}(t-s)} \Phi_{1} \mathcal{F}\left(\Phi_{-1} S(s)\left(\left[\begin{array}{l}
u_{0} \\
v_{0}
\end{array}\right]\right)\right) d s\right\|_{E_{p}^{1} \times E_{p}^{1}} \\
\leq \frac{M_{p} \hat{c}}{\omega}\left(1+\sup _{\left[\begin{array}{c}
u_{0} \\
v_{0}
\end{array}\right] \in \gamma^{+}(B)}\left\|u_{0}\right\|_{E_{2}^{\frac{1}{2}}}^{\rho}\right) .
\end{gathered}
$$

Proof. Recalling (1.16) and Lemma 2.3 we have

$$
\begin{aligned}
\left\|U\left(t,\left[\begin{array}{l}
u_{0} \\
v_{0}
\end{array}\right]\right)\right\|_{E_{2}^{\frac{1+\tilde{\sigma}}{2}} \times E_{2}^{\frac{1+\tilde{\sigma}}{2}}} & =\left\|\Phi_{-1-\frac{\tilde{\sigma}}{2}} \Phi_{1} \int_{0}^{t} e^{-\mathcal{A}_{\mu}(t-s)} \mathcal{F}\left(\Phi_{-1} S(s)\left(\left[\begin{array}{c}
u_{0} \\
v_{0}
\end{array}\right]\right)\right) d s\right\|_{E_{2}^{-\frac{1}{2}} \times E_{2}^{-\frac{1}{2}}} \\
& =\left\|\Phi_{-\frac{\tilde{\sigma}}{2}} \int_{0}^{t} e^{-\mathcal{A}_{\mu}(t-s)} \mathcal{F}\left(\Phi_{-1} S(s)\left(\left[\begin{array}{c}
u_{0} \\
v_{0}
\end{array}\right]\right)\right) d s\right\|_{E_{2}^{-\frac{1}{2}} \times E_{2}^{-\frac{1}{2}}}
\end{aligned}
$$

We remark that $\Phi_{\frac{\tilde{\sigma}}{2}} \in L\left(E_{2}^{-\frac{1}{2}} \times E_{2}^{-\frac{1}{2}}\right)$ and $\Phi_{\frac{\tilde{\sigma}}{2}}$ commutes with $\mathcal{A}_{\mu}$ and hence with $e^{-\mathcal{A}_{\mu} t}$. Thus $\Phi_{\frac{\tilde{\sigma}}{2}}^{-1}=\Phi_{-\frac{\tilde{\sigma}}{2}}$ commutes with $e^{-\mathcal{A}_{\mu} t}$ on $E_{2}^{\frac{\tilde{\sigma}-1}{2}} \times E_{2}^{\frac{\tilde{\sigma}-1}{2}}$ and from (3.3) we get

$$
\begin{aligned}
& \left\|U\left(t,\left[\begin{array}{c}
u_{0} \\
v_{0}
\end{array}\right]\right)\right\|_{E_{2}^{\frac{1+\tilde{\sigma}}{2}} \times E_{2}^{\frac{1+\tilde{\sigma}}{2}}}=\left\|\int_{0}^{t} e^{-\mathcal{A}_{\mu}(t-s)} \Phi_{-\frac{\tilde{\sigma}}{2}} \mathcal{F}\left(\Phi_{-1} S(s)\left(\left[\begin{array}{c}
u_{0} \\
v_{0}
\end{array}\right]\right)\right) d s\right\|_{E_{2}^{-\frac{1}{2}} \times E_{2}^{-\frac{1}{2}}} \\
& \leq \int_{0}^{t}\left\|e^{-\mathcal{A}_{\mu}(t-s)}\right\|_{L\left(E_{2}^{-\frac{1}{2}} \times E_{2}^{-\frac{1}{2}}\right)}\left\|\Phi_{-\frac{\tilde{\sigma}}{2}} \mathcal{F}\left(\Phi_{-1} S(s)\left(\left[\begin{array}{c}
u_{0} \\
v_{0}
\end{array}\right]\right)\right)\right\|_{E_{2}^{-\frac{1}{2}} \times E_{2}^{-\frac{1}{2}}} d s .
\end{aligned}
$$

Since via Theorem 1.2 ii) the positive orbit $\gamma^{+}(B)$ is bounded in $E_{2}^{\frac{1}{2}} \times E_{2}^{\frac{1}{2}}$, Lemma 3.1 implies

$$
\begin{aligned}
\left\|\Phi_{-\frac{\tilde{\sigma}}{2}} \mathcal{F}\left(\Phi_{-1} S(s)\left(\left[\begin{array}{c}
u_{0} \\
v_{0}
\end{array}\right]\right)\right)\right\|_{E_{2}^{-\frac{1}{2}} \times E_{2}^{-\frac{1}{2}}}=\left\|\mathcal{F}\left(\Phi_{-1} S(s)\left(\left[\begin{array}{c}
u_{0} \\
v_{0}
\end{array}\right]\right)\right)\right\|_{E_{2}^{\frac{\tilde{\sigma}-1}{2}} \times E_{2}^{\frac{\tilde{\sigma}-1}{2}}} \\
\leq \hat{c}\left(1+\sup _{\left[\begin{array}{c}
u_{0} \\
v_{0}
\end{array}\right] \in \gamma^{+}(B)}\left\|u_{0}\right\|_{E_{2}^{\frac{1}{2}}}^{\rho}\right),\left[\begin{array}{c}
u_{0} \\
v_{0}
\end{array}\right] \in B, s \geq 0 .
\end{aligned}
$$

With (3.5) and with the exponential estimate of Lemma 2.2, part $(\alpha)$ now follows easily.

For the proof of part $(\beta)$ we take $\tilde{\sigma}=1$, which is possible thanks to the estimate (3.2). We then replace symbol $E_{2}$ with $E_{p}$ in (3.3)-(3.5) to get

$$
\begin{aligned}
& \left\|U\left(t,\left[\begin{array}{c}
u_{0} \\
v_{0}
\end{array}\right]\right)\right\|_{E_{p}^{1} \times E_{p}^{1}}=\left\|\int_{0}^{t} e^{-\mathcal{A}_{\mu}(t-s)} \Phi_{-\frac{1}{2}} \mathcal{F}\left(\Phi_{-1} S(s)\left(\left[\begin{array}{c}
u_{0} \\
v_{0}
\end{array}\right]\right)\right) d s\right\|_{E_{p}^{-\frac{1}{2}} \times E_{p}^{-\frac{1}{2}}} \\
& \leq \int_{0}^{t}\left\|e^{-\mathcal{A}_{\mu}(t-s)}\right\|_{L\left(E_{p}^{-\frac{1}{2}} \times E_{p}^{-\frac{1}{2}}\right)}\left\|\Phi_{-\frac{1}{2}} \mathcal{F}\left(\Phi_{-1} S(s)\left(\left[\begin{array}{c}
u_{0} \\
v_{0}
\end{array}\right]\right)\right)\right\|_{E_{p}^{-\frac{1}{2}} \times E_{p}^{-\frac{1}{2}}} d s,
\end{aligned}
$$

where, thanks to (3.2), for $\left[\begin{array}{l}u_{0} \\ v_{0}\end{array}\right] \in B$ and $s \geq 0$ we have

$$
\begin{aligned}
\left\|\Phi_{-\frac{1}{2}} \mathcal{F}\left(\Phi_{1}^{-1} S(s)\left(\left[\begin{array}{l}
u_{0} \\
v_{0}
\end{array}\right]\right)\right)\right\|_{E_{p}^{-\frac{1}{2}} \times E_{p}^{-\frac{1}{2}}} & =\left\|\mathcal{F}\left(\Phi_{1}^{-1} S(s)\left(\left[\begin{array}{c}
u_{0} \\
v_{0}
\end{array}\right]\right)\right)\right\|_{E_{p} \times E_{p}} \\
& \leq \hat{c}\left(1+\sup _{\left[\begin{array}{c}
u_{0} \\
v_{0}
\end{array}\right] \in \gamma^{+}(B)}\left\|u_{0}\right\|_{E_{2}^{\frac{1}{2}}}^{\rho}\right) .
\end{aligned}
$$

The result now follows via (2.2). 
Corollary 3.1. Under the assumptions of Lemma 3.2 the semigroup $S(t): H_{0}^{1}(\Omega) \times$ $H_{0}^{1}(\Omega) \rightarrow H_{0}^{1}(\Omega) \times H_{0}^{1}(\Omega), t \geq 0$, is asymptotically compact (equivalently asymptotically smooth).

Proof. Since the embedding $E_{2}^{\frac{1+\tilde{\sigma}}{2}} \hookrightarrow E_{1}^{\frac{1}{2}}$ is compact, thanks to (1.16) and Lemmas 2.2 , 3.2 the semigroup $\{S(t)\}$ is the sum of an exponentially decaying linear semigroup and of a family of maps $U(t)$ compact for $t>0$. The result thus follows from [10, Lemma 3.2.3].

Remark 3.1. Theorem 1.2 and Corollary 3.1 ensure the existence of a global attractor $\mathbf{A}$ in the presence of the assumption (1.8). Indeed, $\{S(t)\}$ is the asymptotically smooth semigroup with bounded orbits of bounded sets whereas the set of equilibria is bounded and attracts points. The result thus follows from [10, Theorem 3.4.6].

A bootstrapping procedure for the solutions in the attractor will lead to the regularity result of Theorem 1.3 . The next four lemmas contain a proof of this fact.

Lemma 3.3. Suppose that the assumptions of Lemma 3.2 hold and let $\mathbf{A}$ denote a global attractor for $\{S(t)\}$ in $H_{0}^{1}(\Omega) \times H_{0}^{1}(\Omega)$ (see Remark 3.1). Then

(a) $\mathbf{A}$ is a bounded subset of $H^{1+\tilde{\sigma}}(\Omega) \cap H_{0}^{1}(\Omega) \times H^{1+\tilde{\sigma}}(\Omega) \cap H_{0}^{1}(\Omega)$ if $N \geq 3$,

(b) A is bounded in $W^{2, q}(\Omega) \cap W_{0}^{1, q}(\Omega) \times W^{2, q}(\Omega) \cap W_{0}^{1, q}(\Omega)$ with any $q \in(1, \infty)$ if $N \in\{1,2\}$.

Proof. Note that $\mathbf{A}$ is invariant and, in particular, any point from $\mathbf{A}$ can be viewed as an $H_{0}^{1}(\Omega) \times H_{0}^{1}(\Omega)$-limit of a sequence of the form

$$
S\left(t_{n}\right)\left(\left[\begin{array}{l}
u_{0 n} \\
v_{0 n}
\end{array}\right]\right)=e^{-\mathcal{A}_{\mu} t_{n}}\left[\begin{array}{l}
u_{0 n} \\
v_{0 n}
\end{array}\right]+U\left(t_{n},\left[\begin{array}{l}
u_{0 n} \\
v_{0 n}
\end{array}\right]\right) \text {, where } t_{n} \nearrow \infty \text { and }\left\{\left[\begin{array}{l}
u_{0 n} \\
v_{0 n}
\end{array}\right]\right\} \subset \mathbf{A} .
$$

Since $\mathbf{A}$ is bounded in $H_{0}^{1}(\Omega) \times H_{0}^{1}(\Omega)$, conditions (a), (b) follow from Lemmas 2.2 , 3.2 and from the weak convergence property.

Lemma 3.4. Under the assumptions of Lemma 3.3, if $N \geq 3$, then $\mathbf{A}$ is bounded in the norm of $H^{2}(\Omega) \cap H_{0}^{1}(\Omega) \times H^{2}(\Omega) \cap H_{0}^{1}(\Omega)$.

Proof. If $\tilde{\sigma}=1$, then the result coincides with Lemma 3.3 (a).

Suppose that $\tilde{\sigma}<1$ and set $r_{0}:=\tilde{\sigma}, r_{1}=\min \left\{1, \frac{N+2}{N-2} r_{0}\right\}$. Since $1<\rho<\frac{N+2}{N-2}=$ $\rho^{*}$, we infer from (1.7) that $|f(s)| \leq \bar{c}\left(1+|s|^{\rho^{*}}\right)$ for $s \in \mathbb{R}$ and hence

$$
\left\|f^{e}((I+A) \phi)\right\|_{E_{2}^{\frac{r_{1}-1}{2}}} \leq \tilde{c}\|f(\phi)\|_{E_{\frac{2 N}{N+2\left(1-r_{1}\right)}}} \leq \hat{c}\left(1+\|\phi\|_{E_{2}^{\frac{1+r_{0}}{2}}}^{\rho^{*}}\right), \phi \in E_{2}^{\frac{1+r_{0}}{2}} .
$$

As a consequence of Theorem 1.3 and Lemma 3.3 the set $\mathbf{A}$ is invariant and bounded in $E_{2}^{\frac{1+r_{0}}{2}} \times E_{2}^{\frac{1+r_{0}}{2}}$, so that (3.8) implies

$$
\begin{aligned}
& \left\|\Phi_{-\frac{r_{1}}{2}} \mathcal{F}\left(\Phi_{-1} S(s)\left(\left[\begin{array}{c}
u_{0} \\
v_{0}
\end{array}\right]\right)\right)\right\|_{E_{2}^{-\frac{1}{2}} \times E_{2}^{-\frac{1}{2}}}=\left\|\mathcal{F}\left(\Phi_{-1} S(s)\left(\left[\begin{array}{l}
u_{0} \\
v_{0}
\end{array}\right]\right)\right)\right\|_{E_{2}^{\frac{r_{1}-1}{2}} \times E_{2}^{\frac{r_{1}-1}{2}}} \\
& \leq \hat{c}\left(1+\sup _{\left[\begin{array}{c}
u_{0} \\
v_{0}
\end{array}\right] \in \mathbf{A}}\left\|u_{0}\right\|_{E_{2}}^{\rho^{*}}{ }^{\frac{1+r_{0}}{2}}\right),\left[\begin{array}{c}
u_{0} \\
v_{0}
\end{array}\right] \in \mathbf{A}, s \geq 0 .
\end{aligned}
$$


Estimating in a similar manner as in Lemma 3.2 we get

$$
\begin{aligned}
& \left\|U\left(t,\left[\begin{array}{c}
u_{0} \\
v_{0}
\end{array}\right]\right)\right\|_{E_{2}^{\frac{1+r_{1}}{2}} \times E_{2}^{\frac{1+r_{1}}{2}}}=\left\|\Phi_{-1-\frac{r_{1}}{2}} \Phi_{1} \int_{0}^{t} e^{-\mathcal{A}_{\mu}(t-s)} \mathcal{F}\left(\Phi_{-1} S(s)\left(\left[\begin{array}{c}
u_{0} \\
v_{0}
\end{array}\right]\right)\right) d s\right\|_{E_{2}^{-\frac{1}{2}} \times E_{2}^{-\frac{1}{2}}} \\
& \leq \int_{0}^{t}\left\|e^{-\mathcal{A}_{\mu}(t-s)}\right\|_{L\left(E_{2}^{-\frac{1}{2}} \times E_{2}^{-\frac{1}{2}}\right)} \| \Phi_{-\frac{r_{1}}{2}} \mathcal{F}\left(\Phi_{-1} S(s)\left(\left[\left[\begin{array}{c}
u_{0} \\
v_{0}
\end{array}\right]\right)\right) \|_{E_{2}^{-\frac{1}{2}} \times E_{2}^{-\frac{1}{2}}} d s\right.
\end{aligned}
$$

and hence (using (2.2) and (3.9))

$$
\sup _{\left[\begin{array}{l}
u_{0} \\
v_{0}
\end{array}\right] \in \mathbf{A}} \sup _{t \geq 0}\left\|U\left(t,\left[\begin{array}{l}
u_{0} \\
v_{0}
\end{array}\right]\right)\right\|_{E_{2}^{\frac{1+r_{1}}{2}} \times E_{2}^{\frac{1+r_{1}}{2}}<\infty}
$$

Recalling that any point from $\mathbf{A}$ can be viewed as an $H_{0}^{1}(\Omega) \times H_{0}^{1}(\Omega)$-limit of a sequence of the form given in (3.7), via weak convergence we infer from (2.2), (3.7), (3.11) that

$$
\sup _{\left[\begin{array}{l}
u_{0} \\
v_{0}
\end{array}\right] \in \mathbf{A}}\left\|\left[\begin{array}{l}
u_{0} \\
v_{0}
\end{array}\right]\right\|_{E_{2}^{\frac{1+r_{1}}{2}} \times E_{2}^{\frac{1+r_{1}}{2}}}<\infty
$$

If $r_{1}=\frac{N+2}{N-2} r_{0}<1$, then we repeat the above procedure and obtain that

$$
\text { A is bounded in } E_{2}^{\frac{1+r_{2}}{2}} \times E_{2}^{\frac{1+r_{2}}{2}} \text {, where } r_{2}=\min \left\{1,\left(\frac{N+2}{N-2}\right)^{2} r_{0}\right\} \text {. }
$$

In a finite number of $k$-steps we will then reach $r_{k}=1$, which gives the result.

Lemma 3.5. Under the assumptions and notation of Lemma 3.3, if $N \geq 3$ and a number $q \in(1, \infty)$ is such that $\mathbf{A}$ is bounded in $E_{q}^{1} \times E_{q}^{1}$, then $\mathbf{A}$ is bounded in $E_{q^{*}}^{1} \times E_{q^{*}}^{1}$, where

$$
\begin{aligned}
& q^{*}=\frac{N}{\rho^{*}} \frac{q}{N-2 q} \text { in the case when } N>2 q, \\
& q^{*} \text { is an arbitrary number from }(1, \infty) \text { when } N \leq 2 q .
\end{aligned}
$$

Proof. For $1<\rho<\frac{N+2}{N-2}=\rho^{*}$ we infer from (1.7) that

$$
\left\|f^{e}((I+A) \phi)\right\|_{E_{q^{*}}} \leq \tilde{c}\|f(\phi)\|_{E_{q^{*}}} \leq \bar{c}\left\|1+|\phi|^{\rho^{*}}\right\|_{E_{q^{*}}} \leq \hat{c}\left(1+\|\phi\|_{E_{q}^{1}}^{\rho^{*}}\right), \phi \in E_{q}^{1} .
$$

Since $\mathbf{A}$ is invariant and bounded in $E_{q}^{1} \times E_{q}^{1}$, (3.15) implies for $\left[\begin{array}{l}u_{0} \\ v_{0}\end{array}\right] \in \mathbf{A}$ and $s \geq 0$,

$$
\begin{aligned}
\left\|\Phi_{-\frac{1}{2}} \mathcal{F}\left(\Phi_{-1} S(s)\left(\left[\begin{array}{c}
u_{0} \\
v_{0}
\end{array}\right]\right)\right)\right\|_{E_{q^{*}}^{-\frac{1}{2}} \times E_{q^{*}}^{-\frac{1}{2}}} & =\left\|\mathcal{F}\left(\Phi_{-1} S(s)\left(\left[\begin{array}{c}
u_{0} \\
v_{0}
\end{array}\right]\right)\right)\right\|_{E_{q^{*}} \times E_{q^{*}}} \\
& \leq \hat{c}\left(1+\sup _{\left[\begin{array}{c}
u_{0} \\
v_{0}
\end{array}\right] \in \mathbf{A}}\left\|u_{0}\right\|_{E_{q}^{1}}^{\rho^{*}}\right) .
\end{aligned}
$$

From this and (2.2) we thus have for a certain constant $D>0$ and all $\left[\begin{array}{l}u_{0} \\ v_{0}\end{array}\right] \in \mathbf{A}$, $t \geq 0$,

$$
\begin{aligned}
& \left\|U\left(t,\left[\begin{array}{c}
u_{0} \\
v_{0}
\end{array}\right]\right)\right\|_{E_{q^{*}}^{1} \times E_{q^{*}}^{1}}=\left\|\Phi_{-1-\frac{1}{2}} \Phi_{1} \int_{0}^{t} e^{-\mathcal{A}_{\mu}(t-s)} \mathcal{F}\left(\Phi_{-1} S(s)\left(\left[\begin{array}{c}
u_{0} \\
v_{0}
\end{array}\right]\right)\right) d s\right\|_{E_{q^{*}}^{-\frac{1}{2}} \times E_{q^{*}}^{-\frac{1}{2}}} \\
& \leq \int_{0}^{t}\left\|e^{-\mathcal{A}_{\mu}(t-s)}\right\|_{L\left(E_{q^{*}}^{-\frac{1}{2}} \times E_{q^{*}}^{-\frac{1}{2}}\right)}\left\|\Phi_{-\frac{1}{2}} \mathcal{F}\left(\Phi_{-1} S(s)\left(\left[\begin{array}{c}
u_{0} \\
v_{0}
\end{array}\right]\right)\right)\right\|_{E_{q^{*}}^{-\frac{1}{2}} \times E_{q^{*}}^{-\frac{1}{2}}} d s \leq D .
\end{aligned}
$$


Now viewing points from $\mathbf{A}$ as $H_{0}^{1}(\Omega) \times H_{0}^{1}(\Omega)$-limits of sequences of the form (3.7) we obtain via (2.2), (3.7) and the weak convergence property that

$$
\sup _{\left[\begin{array}{l}
u_{0} \\
v_{0}
\end{array}\right] \in \mathbf{A}}\left\|\left[\begin{array}{l}
u_{0} \\
v_{0}
\end{array}\right]\right\|_{E_{q^{*}}^{1} \times E_{q^{*}}^{1}}<\infty .
$$

The next simple lemma establishes, together with Lemma 3.5 and Lemma 3.4 the asymptotic bootstrapping procedure.

Lemma 3.6. If $N \geq 3$ and $\rho^{*}=\frac{N+2}{N-2}$, then the sequence $\left\{q_{k}\right\}$ defined as

$$
q_{0}=2, \quad q_{k+1}=\frac{N}{\rho^{*}} \frac{q_{k}}{N-2 q_{k}} \text { if } \frac{N}{2}>q_{k}, \quad q_{k+1}=N \quad \text { if } \frac{N}{2} \leq q_{k},
$$

has the property that $q_{k}=N$ for all $k$ sufficiently large.

Proof. Note that $q_{1} \geq q_{0}$ and hence $q_{k+1} \geq q_{k}$ for all $k \in \mathbb{N}$. If $q_{k}<\frac{N}{2}$ for each $k \in \mathbb{N}$, then we would have $q_{0} \leq \lim q_{n}=\frac{N\left(\rho^{*}-1\right)}{2 \rho^{*}}=2 \frac{N}{N+2}<q_{0}$, which is impossible.

With these, all the conclusions of Theorem 1.3 connected with the subcritical case (1.8) are proved.

\section{Asymptotic smoothness of $\{S(t)\}$ and the attractor IN THE CRITICAL CASE}

This section is devoted to the proof of existence of the attractor for the semigroup $\{S(t)\}$ associated in $H_{0}^{1}(\Omega) \times H_{0}^{1}(\Omega)$ to the evolution problem (1.1)-(1.2) in the critical case (1.9), completing the proof of Theorem 1.3. It only remains to prove the asymptotic compactness of $\{S(t)\}$. To that end we will employ Alekseev's Nonlinear Variation of Constants Formula (see [2, [5]). We first need to prove differentiability of the map $f^{e}: H^{-1}(\Omega) \rightarrow H^{-1}(\Omega)$.

Lemma 4.1. Suppose that $f \in C^{1}(\mathbb{R}, \mathbb{R}), N>p$, and $\left|f^{\prime}(s)\right| \leq c\left(1+|s|^{\frac{2 p}{N-p}}\right)$, $s \in \mathbb{R}$, for some $c>0$. Then the map $f^{e}=f \circ(I+A)^{-1}$ is of the class $C^{1}\left(W^{-1, p}(\Omega), W^{-1, p}(\Omega)\right)$.

Proof. Since $(I+A)^{-1}$ is a linear isomorphism from $W^{-1, p}(\Omega)$ into $W^{1, p}(\Omega)$, it is Fréchet differentiable between these spaces with the derivative $(I+A)^{-1}$. Therefore, it remains to ensure that $f$ is continuously Fréchet differentiable from $W^{1, p}(\Omega)$ into $W^{-1, p}(\Omega)$.

Under our assumptions a sufficient condition for this is

$$
\left\|f^{\prime}(\theta(u+h)+(1-\theta) u)-f^{\prime}(u)\right\|_{L^{\frac{N}{2}}(\Omega)} \rightarrow 0 \text { when }\|h\|_{W^{1, p}(\Omega)} \rightarrow 0,
$$

where $\theta$ varies in the interval $[0,1]$.

Since we know that $\left|f^{\prime}\left(\theta\left(u+h_{n}\right)+(1-\theta) u\right)-f^{\prime}(u)\right|$ tends to zero a.e. in $\Omega$ as $\left\|h_{n}\right\|_{W^{1, p}(\Omega)} \rightarrow 0$, we will apply in what follows the Inverse Dominated Convergence Theorem. As a consequence of this theorem we infer from the fact that $\left\|h_{n}\right\|_{L^{\frac{N p}{N-p}}(\Omega)} \rightarrow 0$ that there exists a subsequence $\left\{n_{k}\right\}$, for which $h_{n_{k}} \rightarrow 0$ a.e. in $\Omega$ and also

$$
\left|h_{n_{k}}(x)\right|^{\frac{N p}{N-p}} \leq|g(x)|^{\frac{N p}{N-p}} \text { for a.e. } x \in \Omega,
$$

with $g$ being a certain element of $L^{\frac{N p}{N-p}}(\Omega)$. 
Fixing $u$ and recalling the growth of $f^{\prime}$ we have a.e. in $\Omega$,

$\left|f^{\prime}\left(\theta\left(u+h_{n_{k}}\right)+(1-\theta) u\right)-f^{\prime}(u)\right|^{\frac{N}{2}} \leq c\left(1+\left|h_{n}\right|^{\frac{N p}{N-p}}+|u|^{\frac{N p}{N-p}}\right) \leq c\left(1+|g|^{\frac{N p}{N-p}}+|u|^{\frac{N p}{N-p}}\right)$, where $u, g \in L^{\frac{N p}{N-p}}(\Omega)$. Condition (4.1) now follows easily.

Next we introduce a decomposition of the nonlinearity $f$ which plays an essential role in the proof of condition (1.6).

Lemma 4.2. Suppose that $f \in C^{1}(\mathbb{R}, \mathbb{R})$ and $\limsup _{|s| \rightarrow \infty} f(s) s^{-1}=\mu \in \mathbb{R}$. Then there are functions $f_{1} \in C^{1}(\mathbb{R}, \mathbb{R})$ and $f_{2}: \mathbb{R} \rightarrow \mathbb{R}$ linear such that $f(s)=$ $f_{1}(s)+f_{2}(s)$ and $s f_{1}(s) \leq 0$ for all $s \in \mathbb{R}$,

Proof. It is sufficient to define $f_{2}(s)=f(0)+K s$, where $K \geq \max \left\{\mu, f^{\prime}(0)\right\}$ is a constant for which $s^{-1}(f(s)-f(0))<K$ for all $s \neq 0$.

A decomposition of the nonlinear term $\mathcal{F}$ and of the semigroup of global solutions of (1.13) can now be established in accordance with the decomposition of $f$.

Lemma 4.3. Suppose that $f \in C^{1}(\mathbb{R}, \mathbb{R}), \lim _{\sup } \sup _{|s| \rightarrow \infty} f(s) s^{-1}=\mu \in \mathbb{R}, N>p$, and $\left|f^{\prime}(s)\right| \leq c\left(1+|s|^{\frac{2 p}{N-p}}\right), s \in \mathbb{R}$, for some $c>0$.

Under these assumptions the following conditions hold.

$(\mathrm{C} 1)$ There are functions $f_{1} \in C^{1}(\mathbb{R}, \mathbb{R})$ and $f_{2}: \mathbb{R} \rightarrow \mathbb{R}$ linear such that $f(s)=$ $f_{1}(s)+f_{2}(s), s f_{1}(s) \leq 0$ for $s \in \mathbb{R}$, and

$$
\begin{aligned}
\mathcal{F}\left(\left[\begin{array}{l}
v \\
w
\end{array}\right]\right)=\left[\begin{array}{c}
0 \\
f_{1}\left((I+A)^{-1} v\right)
\end{array}\right]+\left[\begin{array}{c}
0 \\
f_{2}\left((I+A)^{-1} v\right)
\end{array}\right]=: \mathcal{F}_{1}\left(\left[\begin{array}{c}
v \\
w
\end{array}\right]\right)+\mathcal{F}_{2}\left(\left[\begin{array}{c}
v \\
w
\end{array}\right]\right) \\
\text { for }\left[\begin{array}{c}
v \\
w
\end{array}\right] \in W^{-1, p}(\Omega) \times W^{-1, p}(\Omega), \text { where } \\
\mathcal{F}_{1} \in C^{1}\left(W^{-1, p}(\Omega) \times W^{-1, p}(\Omega), W^{-1, p}(\Omega) \times W^{-1, p}(\Omega)\right), \\
\mathcal{F}_{2} \in C^{1}\left(W^{-1, p}(\Omega) \times W^{-1, p}(\Omega), W^{1, p}(\Omega) \times W^{1, p}(\Omega)\right) .
\end{aligned}
$$

(C2) If $\tilde{T}\left(t,\left[\begin{array}{c}v_{0} \\ w_{0}\end{array}\right]\right)$ solves

and if $T\left(t,\left[\begin{array}{l}v_{0} \\ w_{0}\end{array}\right]\right)$ solves

$$
\frac{d}{d t}\left[\begin{array}{c}
\tilde{w} \\
\tilde{w}
\end{array}\right]+\mathcal{A}_{\mu}\left[\begin{array}{c}
\tilde{v} \\
\tilde{w}
\end{array}\right]=\mathcal{F}_{1}\left(\left[\begin{array}{c}
\tilde{v} \\
\tilde{w}
\end{array}\right]\right), t>0, \quad\left[\begin{array}{c}
\tilde{v} \\
\tilde{w}
\end{array}\right]_{t=0}=\left[\begin{array}{c}
v_{0} \\
w_{0}
\end{array}\right]
$$

$$
\frac{d}{d t}\left[\begin{array}{c}
v \\
w
\end{array}\right]+\mathcal{A}_{\mu}\left[\begin{array}{c}
v \\
w
\end{array}\right]=\mathcal{F}_{1}\left(\left[\begin{array}{c}
v \\
w
\end{array}\right]\right)+\mathcal{F}_{2}\left(\left[\begin{array}{c}
v \\
w
\end{array}\right]\right), t>0, \quad\left[\begin{array}{l}
v \\
w
\end{array}\right]_{t=0}=\left[\begin{array}{c}
v_{0} \\
w_{0}
\end{array}\right]
$$

then

$T\left(t,\left[\begin{array}{c}v_{0} \\ w_{0}\end{array}\right]\right)=\tilde{T}\left(t,\left[\begin{array}{c}v_{0} \\ w_{0}\end{array}\right]\right)+\int_{0}^{t}(\partial \tilde{T})\left(t-s, T(s)\left[\begin{array}{c}v_{0} \\ w_{0}\end{array}\right]\right) \mathcal{F}_{2}\left(T\left(s,\left[\begin{array}{c}v_{0} \\ w_{0}\end{array}\right]\right)\right) d s, \quad t>0$,

where $\partial \tilde{T}$ denotes the partial derivative of the map

$(0, \infty) \times W^{-1, p}(\Omega) \times W^{-1, p}(\Omega) \ni\left(t,\left[\begin{array}{c}v_{0} \\ w_{0}\end{array}\right]\right) \rightarrow \tilde{T}\left(t,\left[\begin{array}{c}v_{0} \\ w_{0}\end{array}\right]\right) \in W^{-1, p}(\Omega) \times W^{-1, p}(\Omega)$

with respect to $\left[\begin{array}{l}v_{0} \\ w_{0}\end{array}\right]$ and

$$
\mathcal{F}_{2}\left(\left[\begin{array}{l}
v \\
w
\end{array}\right]\right)=\left[K(I+A)^{-1} v+f(0)\right], \quad\left[\begin{array}{c}
v \\
w
\end{array}\right] \in W^{-1, p}(\Omega) \times W^{-1, p}(\Omega) .
$$

(C3) Function $(\partial \tilde{T})\left(\cdot,\left[\begin{array}{c}v_{0} \\ w_{0}\end{array}\right]\right) \mathcal{F}_{2}\left(\left[\begin{array}{c}\hat{v}_{0} \\ \hat{w}_{0}\end{array}\right]\right)$ is a mild solution of

$$
\frac{d}{d t} \mathcal{W}+\mathcal{A}_{\mu} \mathcal{W}=\mathcal{F}_{1}^{\prime}\left(\tilde{T}\left(t,\left[\begin{array}{l}
v_{0} \\
w_{0}
\end{array}\right]\right) \mathcal{W}, t>0, \quad \mathcal{W}(0)=\mathcal{F}_{2}\left(\left[\begin{array}{c}
\hat{v}_{0} \\
\hat{w}_{0}
\end{array}\right]\right) .\right.
$$


Proof. Condition (C1) follows from Lemmas 4.1 and 4.2 ,

Since $\mathcal{A}_{\mu}$ can be viewed as a sectorial operator in $W^{-1, p}(\Omega) \times W^{-1, p}(\Omega)$ and (4.3) holds, then (C2) follows from [5, Lemma 7]. For the proof of (C3) we refer to [12, Theorem 3.4.4].

In Lemma 4.4 below we will obtain some suitable estimates for $\tilde{T}(\cdot, \cdot), T(\cdot, \cdot)$ and $\mathcal{W}(\cdot)$.

Lemma 4.4. If (1.4) and (1.9) are satisfied, then Lemma 4.3 applies with $p=2$ and the following properties hold.

$(\mathrm{P} 1) H^{-1}(\Omega) \times H^{-1}(\Omega) \ni\left[\begin{array}{c}v_{0} \\ w_{0}\end{array}\right] \rightarrow \tilde{T}\left(t,\left[\begin{array}{c}v_{0} \\ w_{0}\end{array}\right]\right) \in H^{-1}(\Omega) \times H^{-1}(\Omega)$ (where $t \geq 0$ ) is a $C^{0}$ semigroup of global solutions to (4.4) with bounded (positive) orbits of bounded sets.

(P2) $H^{-1}(\Omega) \times H^{-1}(\Omega) \ni\left[\begin{array}{c}v_{0} \\ w_{0}\end{array}\right] \rightarrow T\left(t,\left[\begin{array}{c}v_{0} \\ w_{0}\end{array}\right]\right) \in H^{-1}(\Omega) \times H^{-1}(\Omega)$ (where $t \geq 0$ ) is a $C^{0}$ semigroup of global solutions to (4.5) with bounded (positive) orbits of bounded sets.

(P3) If $B$ is a bounded subset of $E_{2}^{-\frac{1}{2}} \times E_{2}^{-\frac{1}{2}}$ and $\left[\begin{array}{l}\chi_{1} \\ \chi_{2}\end{array}\right] \in E_{2}^{\frac{\sigma-1}{2}}$ for some $\sigma \in[0,1]$, then there is a constant $M_{B}$ such that

$$
\sup _{s \geq 0} \sup _{\left[\begin{array}{c}
v_{0} \\
w_{0}
\end{array}\right] \in B}\left\|\mathcal{F}_{1}^{\prime}\left(\tilde{T}\left(s,\left[\begin{array}{c}
v_{0} \\
w_{0}
\end{array}\right]\right)\right)\left[\begin{array}{l}
\chi_{1} \\
\chi_{2}
\end{array}\right]\right\|_{E_{2}^{\frac{\sigma-1}{2}} \times E_{2}^{\frac{\sigma-1}{2}}} \leq M_{B}\left\|\left[\begin{array}{l}
\chi_{1} \\
\chi_{2}
\end{array}\right]\right\|_{E_{2}^{\frac{\sigma-1}{2}} \times E_{2}^{\frac{\sigma-1}{2}}} .
$$

(P4) If $B$ is a bounded subset of $E_{2}^{-\frac{1}{2}} \times E_{2}^{-\frac{1}{2}}$, then for a certain constant $N_{B}>0$,

$$
\sup _{s \geq 0} \sup _{s}\left\|\mathcal{F}_{2}\left(T\left(s,\left[\begin{array}{c}
v_{0} \\
w_{0}
\end{array}\right]\right)\right)\right\|_{E_{2} \times E_{2}} \leq N_{B}
$$

(P5) If $\tau>0$ and $B$ is bounded in $E_{2}^{-\frac{1}{2}} \times E_{2}^{-\frac{1}{2}}$, then there is a constant $\mathcal{R}(\tau, B)$ such that for each $\left[\begin{array}{c}\hat{v}_{0} \\ \hat{w}_{0}\end{array}\right] \in\left\{T\left(s,\left[\begin{array}{c}v_{0} \\ w_{0}\end{array}\right]\right), s \geq 0,\left[\begin{array}{c}v_{0} \\ w_{0}\end{array}\right] \in B\right\}=: \hat{B}$ the mild solution $\mathcal{W}$ of (4.7) fulfills the estimate

$$
\sup _{t \in[0, \tau]}\|\mathcal{W}(t)\|_{E_{2} \times E_{2}} \leq \mathcal{R}(\tau, B)
$$

in particular,

$$
\begin{aligned}
& \text { for all }\left[\begin{array}{c}
\hat{v}_{0} \\
\hat{w}_{0}
\end{array}\right] \in \hat{B} \text { the corresponding values of } \mathcal{W}(t) \text { until } \\
& \text { the time } \tau \text { lie in a certain compact subset of } E_{2}^{-\frac{1}{2}} \times E_{2}^{-\frac{1}{2}} \text {. }
\end{aligned}
$$

Proof. Properties (P1), (P2) follow from Theorem 2.1 and properties of the functional $\mathcal{L}$ in Lemma 2.5 which lemma is applicable since both $f$ and $f_{1}$ satisfy (1.4).

For the proof of $(\mathrm{P} 3)$ we first note that $\mathcal{F}_{1}^{\prime}\left(\left[\begin{array}{l}z_{1} \\ z_{2}\end{array}\right]\right)\left[\begin{array}{l}\chi_{1} \\ \chi_{2}\end{array}\right]=\left[\begin{array}{c}0 \\ f_{1}^{\prime}\left((I+A)^{-1} z_{1}\right)(I+A)^{-1} \chi_{1}\end{array}\right]$ and hence

$$
\begin{aligned}
\left\|\mathcal{F}_{1}^{\prime}\left(\left[\begin{array}{c}
z_{1} \\
z_{2}
\end{array}\right]\right)\left[\begin{array}{c}
\chi_{1} \\
\chi_{2}
\end{array}\right]\right\|_{E_{2}^{\frac{\sigma-1}{2}} \times E_{2}^{\frac{\sigma-1}{2}}} & \leq d\left\|f_{1}^{\prime}\left((I+A)^{-1} z_{1}\right)(I+A)^{-1} \chi_{1}\right\|_{E_{\frac{2 N}{N+2(1-\sigma)}}} \\
& \leq d\left\|f_{1}^{\prime}\left((I+A)^{-1} z_{1}\right)\right\|_{E_{\frac{N}{2}}}\left\|(I+A)^{-1} \chi_{1}\right\|_{E_{\frac{2 N}{N-2(1+\sigma)}}} \\
& \leq d\left\|f_{1}^{\prime}\left((I+A)^{-1} z_{1}\right)\right\|_{E_{\frac{N}{2}}}\left\|(I+A)^{-1} \chi_{1}\right\|_{E_{2}^{\frac{1+\sigma}{2}}} \\
& =d\left\|f_{1}^{\prime}\left((I+A)^{-1} z_{1}\right)\right\|_{E_{\frac{N}{2}}}\left\|\chi_{1}\right\|_{E_{2}^{\frac{\sigma-1}{2}}}
\end{aligned}
$$


With the aid of (1.9) we next obtain

$$
\begin{aligned}
& \left\|\mathcal{F}_{1}^{\prime}\left(\left[\begin{array}{l}
z_{1} \\
z_{2}
\end{array}\right]\right)\left[\begin{array}{l}
\chi_{1} \\
\chi_{2}
\end{array}\right]\right\|_{E_{2}^{\frac{\sigma-1}{2}} \times E_{2}^{\frac{\sigma-1}{2}}} \leq d\left\|c\left(1+\left|(I+A)^{-1} z_{1}\right|^{\frac{4}{N-2}}\right)\right\|_{E_{\frac{N}{2}}}\left\|\chi_{1}\right\|_{E_{2}^{\frac{\sigma-1}{2}}} \\
& \leq c d\left(\|1\|_{E_{\frac{N}{2}}}+\left\|(I+A)^{-1} z_{1}\right\|_{E_{\frac{2 N}{N-2}}^{\frac{4}{N-2}}}^{\frac{2}{N-2}}\right)\left\|\chi_{1}\right\|_{E_{2}^{\frac{\sigma-1}{2}}} \leq \tilde{c}\left(1+\left\|z_{1}\right\|_{E_{2}^{-\frac{1}{2}}}^{\frac{4}{N-2}}\right)\left\|\chi_{1}\right\|_{E_{2}^{\frac{\sigma-1}{2}}} .
\end{aligned}
$$

Property (P3) now follows from (P1) and (4.12).

In the proof of $(\mathrm{P} 4)$ we note that $f_{2}(s)=f(0)+K s$ is a linear function and therefore

$$
\left\|\mathcal{F}_{2}\left(\left[\begin{array}{l}
\chi_{1} \\
\chi_{2}
\end{array}\right]\right)\right\|_{E_{2} \times E_{2}}=\left\|f(0)+K(I+A)^{-1} \chi_{1}\right\|_{E_{2}} \leq \tilde{d}\left(1+\left\|\chi_{1}\right\|_{E_{2}^{-\frac{1}{2}}}\right) .
$$

Thus (P4) follows from (P2) and 4.13).

To complete the proof we write the variation of constants formula for $\mathcal{W}$ :

$$
\mathcal{W}(t)=e^{-\mathcal{A}_{\mu} t} \mathcal{F}_{2}\left(\left[\begin{array}{c}
\hat{v}_{0} \\
\hat{w}_{0}
\end{array}\right]\right)+\int_{0}^{t} e^{-\mathcal{A}_{\mu}(t-s)} \mathcal{F}_{1}^{\prime}\left(\tilde{T}\left(s,\left[\begin{array}{c}
v_{0} \\
w_{0}
\end{array}\right]\right)\right) \mathcal{W}(s) d s,
$$

and apply $\Phi_{-\frac{1}{2}}$ to get, with the aid of (P3), (P4) and (2.2),

$$
\begin{aligned}
\|\mathcal{W}(t)\|_{E_{2} \times E_{2}} & =\left\|\Phi_{-\frac{1}{2}} \mathcal{W}(t)\right\|_{E_{2}^{-\frac{1}{2}} \times E_{2}^{-\frac{1}{2}}} \leq\left\|e^{-\mathcal{A}_{\mu} t} \Phi_{-\frac{1}{2}} \mathcal{F}_{2}\left(\left[\begin{array}{c}
\hat{v}_{0} \\
\hat{w}_{0}
\end{array}\right]\right)\right\|_{E_{2}^{-\frac{1}{2}} \times E_{2}^{-\frac{1}{2}}} \\
& +\int_{0}^{t}\left\|e^{-\mathcal{A}_{\mu}(t-s)}\right\|_{E_{2}^{-\frac{1}{2}} \times E_{2}^{-\frac{1}{2}}}\left\|\Phi_{-\frac{1}{2}} \mathcal{F}_{1}^{\prime}\left(\tilde{T}\left(s,\left[\begin{array}{c}
v_{0} \\
w_{0}
\end{array}\right]\right)\right) \mathcal{W}(s)\right\|_{E_{2}^{-\frac{1}{2}} \times E_{2}^{-\frac{1}{2}}} d s \\
& \leq M_{2} e^{-\omega t} N_{B}+\int_{0}^{t} M_{2} e^{-\omega(t-s)}\left\|\mathcal{F}_{1}^{\prime}\left(\tilde{T}\left(s,\left[\begin{array}{c}
v_{0} \\
w_{0}
\end{array}\right]\right)\right) \mathcal{W}(s)\right\|_{E_{2} \times E_{2}} d s \\
& \leq M_{2} N_{B}+\int_{0}^{t} M_{2} M_{B}\|\mathcal{W}(s)\|_{E_{2} \times E_{2}} d s
\end{aligned}
$$

Relation (4.10) is now a consequence of Gronwall's inequality and (4.11) follows via compact embedding $E_{2} \hookrightarrow E_{2}^{-\frac{1}{2}}$.

To conclude the proof of asymptotic compactness for $\{S(t)\}$ it only remains to prove that $\{\tilde{T}(t)\}$ decays exponentially, uniformly in bounded sets.

Lemma 4.5. Given $r>0$ there are constants $c(r)>0$ and $\beta(r)>0$ such that

$$
\sup \left\{\left\|\tilde{T}\left(t,\left[\begin{array}{c}
v_{0} \\
w_{0}
\end{array}\right]\right)\right\|_{E_{2}^{-\frac{1}{2}} \times E_{2}^{-\frac{1}{2}}}:\left\|\left[\begin{array}{c}
v_{0} \\
w_{0}
\end{array}\right]\right\|_{E_{2}^{-\frac{1}{2}} \times E_{2}^{-\frac{1}{2}}} \leq r\right\} \leq c(r) e^{-\beta(r) t} .
$$

Proof. The proof follows as in Proposition 3 of [5] using the functional

$$
\mathcal{L}_{\delta}\left(v, v_{t}\right)=\mathcal{L}\left(v, v_{t}\right)+\delta\left\langle v, v_{t}\right\rangle_{E_{2}^{-\frac{1}{2}}} .
$$

In fact, let

$$
\left[\begin{array}{c}
\tilde{v} \\
\tilde{v}_{t}
\end{array}\right]=: \tilde{T}\left(t,\left[\begin{array}{c}
v_{0} \\
w_{0}
\end{array}\right]\right), \quad t \geq 0 \quad \text { and } \quad\left\|\left[\begin{array}{c}
v_{0} \\
w_{0}
\end{array}\right]\right\|_{E_{2}^{-\frac{1}{2}} \times E_{2}^{-\frac{1}{2}}} \leq r
$$


Choosing $\delta>0$ suitably small we have that

$$
\begin{aligned}
\frac{d}{d t}\left(\mathcal{L}_{\delta}\left(v, v_{t}\right)\right) & \leq-\left(\frac{\mu \lambda_{1}}{1+\lambda_{1}}+\delta\right)\left\|v_{t}\right\|_{E_{2}^{-\frac{1}{2}}}^{2}+\delta\left\langle v,-\mu \Lambda v_{t}-\Lambda v+f_{1}^{e}(v)\right\rangle_{E_{2}^{-\frac{1}{2}}} \\
& \leq-\left(\frac{\mu \lambda_{1}}{1+\lambda_{1}}+\delta\right)\left\|v_{t}\right\|_{E_{2}^{-\frac{1}{2}}}^{2}-\delta\left\langle v, \mu \Lambda v_{t}\right\rangle_{E_{2}^{-\frac{1}{2}}}-\delta\langle v, \Lambda v\rangle_{E_{2}^{-\frac{1}{2}}} \\
& \leq-\frac{\mu \lambda_{1}}{2+2 \lambda_{1}}\left\|v_{t}\right\|_{E_{2}^{-\frac{1}{2}}}^{2}-\frac{\delta}{2}\|v\|_{E_{2}^{-\frac{1}{2}}} .
\end{aligned}
$$

On the other hand, using (2.11) we obtain, for suitably small $\delta$, that

$$
\begin{aligned}
\mathcal{L}_{\delta}\left(v, v_{t}\right)= & \frac{1}{2}\left\|v_{t}\right\|_{E_{2}^{-\frac{1}{2}}}^{2}+\frac{1}{2}\|v\|_{E_{2}^{-\frac{1}{2}}}^{2}-\frac{1}{2}\left\|(I+A)^{-\frac{1}{2}} v\right\|_{E_{2}^{-\frac{1}{2}}}^{2}+\delta\left\langle v, v_{t}\right\rangle_{E_{2}^{-\frac{1}{2}}} \\
& -\int_{\Omega} F_{1}\left((I+A)^{-1} v\right) d x \\
\leq & \frac{1}{2}\left\|v_{t}\right\|_{E_{2}^{-\frac{1}{2}}}^{2}+\frac{1}{2}\|v\|_{E_{2}^{-\frac{1}{2}}}^{2}+\delta\left\langle v, v_{t}\right\rangle_{E_{2}^{-\frac{1}{2}}}+\hat{c}\left(1+\|v\|_{E_{2}^{-\frac{1}{2}}}^{\rho^{*}-1}\|v\|_{E_{2}^{-\frac{1}{2}}}^{2}\right. \\
\leq & \bar{c}(r)\left(\left\|v_{t}\right\|_{E_{2}^{-\frac{1}{2}}}^{2}+\|v\|_{E_{2}^{-\frac{1}{2}}}^{2}\right) .
\end{aligned}
$$

The proof of the result now follows easily.

\section{REFERENCES}

[1] H. Amann, Linear and Quasilinear Parabolic Problems, Birkhäuser, Basel, 1995. MR1345385 (96g:34088)

[2] J. M. Arietta, A. N. Carvalho, J. K. Hale, A damped hyperbolic equation with critical exponent, Comm. Partial Differential Equations 17 (1992), 841-866. MR.1177295(93f:35145)

[3] J. M. Arrieta, A. N. Carvalho, A. Rodriguez-Bernal, Attractors of parabolic problems with nonlinear boundary conditions. Uniform bounds, Comm. Partial Differential Equations 25 (2000), 1-37. MR:1737541 (2001b:35037)

[4] I. L. Bogolubsky, Some examples of inelastic soliton interaction, Comput. Phys. Comm. 13 (1977), 149-155.

[5] A. N. Carvalho, J. W. Cholewa, Attractors for strongly damped wave equations with critical nonlinearities, Pacific J. Math. 207 (2002), 287-310. MR.1972247(2004b:35023)

[6] P. A. Clarkson, R. J. Leveque, R. A. Saxton, Solitary wave interaction in elastic rods, Stud. Appl. Math. 75 (1986), 95-122. MR859173 (87j:73034)

[7] K.-J. Engel, R. Nagel, One-Parameter Semigroups for Linear Evolution Equations, Springer, New York, 2000. MR1721989(2000i:47075)

[8] J.-M. Ghidaglia, A. Marzocchi, Longtime behaviour of strongly damped wave equations, global attractors and their dimension, SIAM J. Math. Anal. 22 (1991), 879-895. MR 1112054 (92e:35033)

[9] D. Gilbarg, N. Trudinger, Elliptic Partial Differential Equations of Second Order, Springer, Berlin, 1983. MR737190 (86c:35035)

[10] J. K. Hale, Asymptotic Behavior of Dissipative Systems, Mathematical Surveys and Monographs, vol. 25, AMS, Providence, RI, 1988. MR941371 (89g:58059)

[11] J. K. Hale and G. Raugel, Convergence in Gradient-Like Systems with Applications to PDE, Z. Angew. Math. Phys. 43 (1992), 63-124. MR1149371(93h:58143)

[12] D. Henry, Geometric Theory of Semilinear Parabolic Equations, Springer-Verlag, Berlin, 1981. MR610244 (83j:35084)

[13] O. A. Ladyženskaya, Attractors for Semigroups and Evolution Equations, Cambridge University Press, Cambridge, 1991. MR,1133627 (92k:58040)

[14] Q. F. Ma, S. H. Wang, C. K. Zhong, Necessary and sufficient conditions for the existence of global attractors for semigroups and applications, Indiana Univ. Math. J. 51 (2002), 15411559. MR 1948459 (2003j:37137) 
[15] A. Pazy, Semigroups of Linear Operators and Applications to Partial Differential Equations, Springer-Verlag, New York, 1983. MR.710486 (85g:47061)

[16] C. E. Seyler, D. L. Fanstermacher, A symmetric regularized long wave equation, Phys. Fluids 27 (1984) 58-66.

[17] Y. D. Shang, Initial-boundary value problem for the equation $u_{t t}-\Delta u-\Delta u_{t}-\Delta u_{t t}=f(u)$, Acta Math. Appl. Sinica (Chinese Ser.) 23 (2000), 385-393. MR1797635 (2001k:35221)

[18] G. Webb, Compactness of bounded trajectories of dynamical systems in infinite-dimensional spaces, Proc. Roy. Soc. Edunburgh Sect. A 84 (1979), 19-33. MR.549869 (80j:34084)

[19] Yongqin Xie, Chengkui Zhong, The existence of global attractors for a class nonlinear evolution equation, J. Math. Anal. Appl. 336 (2007), 54-69. MR.2348490

[20] H. W. Zhang, Q. Y. Hu, Existence of global weak solution and stability of a class nonlinear evolution equation, Acta Math. Sci. 24A (2004), 329-336. MR2072406 (2005d:35180)

[21] C. K. Zhong, M. H. Yang, C. Y. Sun, The existence of global attractors for the normto-weak continuous semigroup, J. Differential Equations 223 (2006), 367-399. MR2214940 (2006k:37221)

[22] W. G. Zhu, Nonlinear waves in elastic rods, Acta Solid Mech. Sinica 1 (1980), 247-253.

Departamento de Matemática, Instituto de Ciências Matemáticas e de ComputaÇão, Universidade de São Paulo-Campus de São Carlos, Caixa Postal 668, 13560-970 São CARlos SP, Brazil

E-mail address: andcarva@icmc.usp.br

Institute of Mathematics, Silesian University, 40-007 Katowice, Poland

E-mail address: jcholewa@ux2.math.us.edu.pl 